\title{
VIII.
}

Aus der Klinik des Professors Maixner in Prag.

\section{Beitrag zur Erklärung der ophthalmoplegischen Migräne.}

Von

\section{Dr. Václar Plavec,}

klinischer Assistent.

Die ophthalmoplegische Migräne ist, klinisch genommen, dieselbe Affektion, welche Möbius, Senator, Karplus u. a. unter der Bezeichnung der periodischen Oculomotoriuslähmung beschreiben. Möbius ${ }^{1}$ ) vermeidet absichtlich die Bezeichnung Migräne, weil er sich vorstellt, dass die Migräne, welche die Lähmungsanfälle begleitet und der ganzen Krankheit den periodischen Charakter verleiht, hier nur ein Symptom einer bestimmten Hirnläsion darsteillt, welche zugleich die Lähmung verursacht. Dagegen hält Charcot $^{2}$ ) und mit ihm vielleicht die ganze französische Schule die Migräne für eine echte und primäre Erkrankung und wendet daher auch die Bezeichnung ophthalmoplegische Migräne an.

Eine ganz ähnliche Ansicht wie Charcot hat Vissering ${ }^{3}$ ) und später auch Oppenheim ${ }^{4}$ ) ausgesprochen, indem sie bei der Oculomotoriuslähmung eine besondere, selbständige Erkrankung annehmen, die in ihrem Wesen der echten Migräne sehr ähnlich. aber mit ihr doch nicht identisch ist. Eine Mittelstellung zwischen der Ansicht von Möbius und Charcot haben in letzter Zeit Karplus ${ }^{5}$ ) und Seiffer ${ }^{6}$ ) eingenommen, indem sie behaupten, es gebe Fälle sowohl der einen als auch der anderen Art, nämlich solche, wo die Migräne eive idiopathische und primäre, und solche, wo sie eine symptomatische resp. sekundäre sei.

Im Gegensatz zu allen diesen Autoren, welche den klinischen Hauptcharakter der ophthalmoplegischen Migräne, resp. der periodischen Oculomotoriuslähmung in dem typischen Migräneanfall erblicken, giebt es andererseits noch Autoren, welche zwar zugeben, dass die periodische Oculomotoriuslähmung durch den Charakter ihrer Anfälle manchmal 
der echten Migräne ähnlich sei, im allgemeinen aber der Ansicht sind, dass die Diagnose der periodischen Oculomotoriuslähmung keineswegs den Migränetypus erfordert, und die die periodische Oculomotoriuslähmung mit der gewöhnlichen rezidivierenden Oculomotoriuslähmung vollkommen indentifizieren (Marina ${ }^{9}$ ), Schmidt-Rimpler ${ }^{7}$ ), Mingazzini $^{9}$ ), Bernheimer ${ }^{8}$ ), Wilbrand und Saenger ${ }^{9}$ ) u. m. a.). Sie zählen hierher auch jene Fälle, wo sich die Anfälle in unregelmässigen, eventuell auch mehrere Jahre betragenden Perioden wiederholten, wo die Kopfschmerzen diffus waren, eventuell mehrere Wochen dauerten, oder aber auch vollständig fehlten.!

Es ist sicherlich ein grosser Unterschied, ob sich die Oculomotoriuslähmung 2 mal oder $3 \mathrm{mal}$ in einem gewissen Lebensalter wiederholt, oder ob sie durch mehrere Jahrzehnte jährlich 10 mal oder auch 20 mal in mehr oder weniger typischen Anfällen wiederkehrt. Klinisch und anatomisch wurde öfters nachgewiesen, dass die einfache, nur einige Male rezidivierende Oculomotoriuslähmung zwar eine gewisse Zeit lang scheinbar selbständig auftreten kann, im übrigen aber durch verschiedene Gehirnkrankheiten bedingt ist (Tumor, Tabes, progressive Paralyse, Lues, Pachymeningitis, Tuberkulose, Neuritis u. a.). Die echte periodische Oculomotoriuslähmung, wie sie Charcot*), speziell aber Möbius beschreibt, ist eine Krankheit von mehr oder weniger einheitlichem Charakter und höchstwahrscheinlich auch einheitlicher Pathogenese, die, mag nun die sie begleitende Migräne eine primäre oder sekundäre sein, in einem jeden Falle durch ihre Anfälle der idiopathischen Migräne sehr ähnlich ist.

Daher ist es nicht richtig, die rezidivierende Lähmung mit der periodischen $\mathrm{zu}$ identifizieren und beide Bezeichnungen promiscue zu verwenden. Auf diese Weise könnten wir uns allerdings erklären, warum das klinische Bild der periodischen Oculomotoriuslähmung bei den einzelnen Autoren kein einheitliches ist, warum es nicht in den Rahmen der Migräne passt. Übrigens ist die rezidivierende Oculomotoriuslähmung selten vollständig rein, sondern in der Mehrzahl der Fälle zeigen sich entweder schon beim ersten Anfall der Lähmung oder erst bei einem späteren Rezidive neben der Oculomotoriuslähmung auch andere Gehirnsymptome (Lähmung der übrigen Augennerven oder auch der anderen Gehirnnerven, Übergang auf die andere Seite, Hemiparese, Krämpfe, Bewusstlosigkeit u. dergl.).

*) Der zweite Fall Charcots (1. c. oder auch Progrès medical 1890 Nr. 31. 32) ist nicht gerade typisch; selbst Schmidt-Rimpler bezeichnet diesen Fall als eine Migräne, welche nachträglich, auf der Basis einer anderen Ätiologie, mit einer rezidivierenden Oculomotoriuslähmung kompliziert wurde. 
Dagegen lässt die echte, rein periodische Oculomotoriuslähmung alle ähnlichen Nebenerscheinungen vermissen, mag sie auch das ganze Leben hindurch dauern. Die einzelnen Anfälle zeichnen sich durch eine grosse Äbnlichkeit aus und beginnen stets mit einem echten Migräneanfall, zu dem sich erst später die Oculomotoriuslähmung gesellt. Ergriffen ist stets nur der eine Oculomotorius und zwar stets auf derselben Seite. Auf andere Nerven greift die Paralyse nicht über, nicht einmal dann, wenn dieselbe ihren Höhepunkt erreicht hat, indem die gesamte von dem betreffenden Oculomotorius versorgte Muskulatur (inklusive der innern) dauernd gelähmt ist, wenngleich die Migräneanfälle sich in gleicher Anzahl und ron gleicher Dauer weiter einstellen (Seiffer).

Gewöhnlich wird beim ersten Anfall nicht gleich der ganze Oculomotorius gelähmt, sondern es zeigt sich nur eine Parese eines Muskels oder zweier Muskeln, die nach dem Anfall gewöhnlich in einigen wenigen Tagen wieder verschwindet. Bei den folgenden Anfällen nimmt die Parese im Laufe der Jahre an Intensität und Ausdehnung allmählich zu, bis bei einem Anfall alle Muskeln des betreffenden Auges, die äussern sowohl als auch die innern, ergriffen sind und die Parese eventuell zugleich in eine totale Paralyse übergeht. Die Reihenfolge, in welcher die Muskulatur ergriffen wird, ist keine bestimmte und schwankt von Fall zu Fall. Einmal beginnt die ophthalmoplegische Migräne mit Ptose und erst später erkrankt die bulbäre Muskulatur, ein ander Mal ist das Gegenteil der Fall, indem die Ptose in dem Bilde der periodischen Lähmung zuletzt eintritt. Was die bulbäre Muskulatur anbelangt, leidet das eine Mal mehr die innere, das andere Mal mehr die äussere Muskulatur. Im Falle $\nabla$ isserings z. B. war die Pupillenreaktion nur wenig gestört, selbst wenn bei einem starken Anfalle alle übrige Muskulatur des Oculomotorius gelähmt war.

Die vollständige Paralyse erfordert zu ihrer Reparation eine längere Dauer und da kann es bei dem progressiven Verlauf dieser Krankheit einmal geschehen, dass ein folgender Anfall früher eintritt, bevor noch die vollständige Restitutio ad integrum stattgefunden hat, wodurch die ursprünglich rein periodische Lähmung (resp. Parese) zu einer periodisch exazerbierenden wird.

Die Anfälle der Migräne und Lübmung können zwar in Bezug auf ihre Intensität Schwankungen aufweisen, aber die Reihenfolge der einzelnen Symptome bei einem und demselben Patienten bleibt dieselbe. Nach einem schweren Anfall mit vollkommener Lähmung kann wiederum ein leichterer Anfall kommen, wo sich eventuell nur die Migräne einstellt oder wo die Oculomotoriusparese kaum angedeutet 
ist. Diese abortiven Anfälle sind auch von kürzerer Dauer. Manchmal besteht anfaigs mehrere Jabre hindurch nur eine einfache, aber stets auch nur einseitige Migräne, während sich die Lähmung erst später hinzugesellt.

Die freien Intervalle żwischen den einzelnen Anfällen sind manchmal fast regelmässig, manchmal wiederum schwanken sie mehr.oder weniger, im allgemeinen pflegt aber die Zahl der Anfälle während eines längeren Zeitraums - eines Jahres - stets annähernd die gleiche zu sein. Die typischen Fälle pflegen gewöhnlich eine grössere Anzahl von Anfällen (5-20) jährlich aufzuweisen.

Aus einem Anfall oder aus drei Anfällen kann die Diagnose auf ophthalmoplegische Migräne nicht gestellt werden, selbst wenn die Anfälle im übrigen typisch sind; handelt es sich aber um eine schon aus dem zarten Kindesalter oder wenigstens aus der Jugend (durch mehrere hinter einander folgende Jahre) sich datierende Krankheit, dann ist die Diagnose leicht nnd sicher. Möbius nimmt als äusserste Grenze, bis zu welcher die echte periodische Oculomotoriuslähmung auftreten kann, etwa das 25. Lebensjahr an. Wenn auch dieser Angabe keine absolute Bedeutung zukommen kann, so ist doch wenigstens so viel sicher, dass die ophthalmoplegische Migräne im späteren Alter sehr selten beginnt.

Unter allen Fällen, die als ophthalmoplegische Migräne beschrieben werden (im ganzen etwa 50), fand ich nur 5, in denen sich der erste Lähmungsanfall nach dem 25. Lebensjahre eingestellt hatte, bei Charcot im 30., bei Chabbert ${ }^{11}$ ) im 51., bei Seiffer im 39., bei Strzeminski11) im 35. und bei $\mathrm{Ryba}^{10}$ ) im 44. Lebensjahre. Alle diese Fälle litten schon in jüngeren Jahren entweder an einer gewöhnlichen oder an einer ophthalmischen Migräne. Aber die Oculomotoriuslähmung besass nur in zwei Fällen einen gut ausgeprägten, mit den Migräneanfällen eng zusammenhängenden periodischen Charakter (Seiffer, Strzeminski). In den übrigen Fällen besassen die die Lähmung begleitenden Kopfschmerzen nicht den echten Migränecharakter, denn sie dauerten mehrere Wochen und waren von einander durch unregelmässige, manchmal sogar mehrere Jahre betragende Intervalle getrennt. Möbius bezeichnet derartige Fälle als rezidivierende Oculomotoriuslähmung bei Migräne und zwar mit Recht, da z. B. die Lähmung im Falle Chabberts später auch das andere Auge ergriff.

Auf derartige rezidivierende Lähmungen bei Migräne muss man übrigens auch bei jüngeren Patienten bedacht sein, da weder die Migräne, noch die organische Oculomotoriuslähmung so seltene Erkrankungen sind, dass eine zufällige Kombination derselben ausgeschlossen werden könnte. 
Obwohl die Oculomotoriuslähmung in derartigen Fällen eigentlich durch eine organische Affektion bedingt ist, die sich unabhängig von der Migräne entwickelt hat, so ist es unter gewissen Dmständen dennoch möglich, dass sie sich im Migräneanfall (d. i. infolge einer Alteration des Blutkreislaufs u. dgl.) verschlimmert, wodurch ein der echten ophthalmoplegischen Migräne sehr ähnliches klinisches Bild entstehen kann, dessen Differentialdiagnose von der echten ophthalmoplegischen Migräne sehr schwierig sein kann (Möbius). Solange wir aber keine besseren klinischen Kriterien besitzen, wird es besser sein, die zweifelhaften Fälle nicht zu der periodischen Migräne zu zählen, damit ihr klinisches Bild ein typisches bleibe. Nur auf diese Weise werden wir zu einer richtigen Klassifikation und zu einer richtigen Erklärung dieser Krankheit gelangen (Möbius, Mauthner ${ }^{11}$ ), Ballet ${ }^{11}$ ), Seiffer, Leclézio'11) u. a.).

Die Zahl der reinen, wirklich typischen Fälle von ophthalmoplegischer Migräne, bei denen seit der Kindheit eine periodische Wiederkehr der Kopfschmerzen und der Oculomotoriuslähmung eine lange Reihe von Jahren hindurch, manchmal bis ins Alter bestand, beträgt in der Literatur ungefähr 30 . Aber bis jetzt ist noch jeder neue Fall dieser Art interessant, da nur aus einer auf vielen Fällen basierenden Statistik die Selbständigkeit dieser Krankheit besser hervortritt.

Bei uns hat vor mehreren Jahren Ryba ${ }^{12}$ ) einen typischen Fall bei einem 23jährigen Mädchen beschrieben, bei dem die Migräneanfälle, die gleich im Anfang von der Ophthalmoplegie begleitet waren, im Alter von $3^{1 / 2}$ Jahren begonnen haben. Bis zum 18. Lebensjahre gingen die Symptome der Oculomotoriuslähmung nach jedem Anfall zurück, aber seit dieser Zeit blieb die Parese auch nach dem Anfall bestehen und nahm mit der Zeit zu. Die Kopfschmerzen dauerten 1-3 Tage, die freien Intervalle gewöhnlich 14 Tage bis 3 Monate (ausnahmsweise auch 9 und einmal sogar 11 Monate).

Einen auffallend ähnlichen Fall beobachteten wir zu Ende des verflossenen und zu Anfang des heurigen Jahres an der Klinik des Herrn Prof. Maixner. Es handelte sich wiederum um ein Mädchen und zwar im Alter von 20 Jahren, dessen Vater Folgendes angab:

Die Mutter der Patientin starb an einer unbekannten Krankheit; 2 Schwestern sind gesund, 4 Brüder starben, davon 3 im zarten Alter, und der vierte nach einem Unfall. Weder der Vater noch die Mutter litten an Migräne. Die Patientin selbst hat nie eine schwere. Krankheit durchgemacht und auch keinen Unfall erlitten.

Die ersten Anfänge der gegenwärtigen Krankheit zeigten sich schon im zarten Alter von ungefähr 2 Jahren. Schon damals beobachteten die 
Eltern, dass das Kind von Zeit zu Zeit, etwa einmal binnen mehrerer (4-6) Wochen, verdriesslich war, weinte, nichts ass und gewöhnlich auch ein- oder mehrmals erbrach. Dieser Zustand dauerte ungefähr einen Tag, worauf dann wieder Wohlbefinden eintrat. Später, als das Kind bereits sprechen konnte, klagte es bei einem jeden derartigen Anfall über Kopfschmerzen. Während der ersten 3 Jahre beobachteten die Eltern sonst nichts Auffallendes. Als aber das Kind die Schule zu besuchen anfing, da steigerten sich die Anfälle, wiederholten sich manchmal schon nach 14 Tagen und die Kopfschmerzen dauerten 2-3 Tage. Da erst fiel es den Eltern auf, dass das linke Auge im Anfalle, wenn dieser etwa 2 Tage gedauert hatte, nach aussen und etwas nach unten verdreht wurde. Damals zeigten die Lider dieses Auges noch nichts Besonderes. Nach einem jeden Anfall ging die Parese am linken Auge binnen einigen Tagen zurück und die Patientin fühlte sich überhaupt vollkommen gesund. Nur bemerkte sie, dass sie beim Lernen in der Schule auf dem linken Auge weniger gut sah, was auch der Arzt damals sofort konstatiert hatte. Die Sehstörung hat sich infolge des Anfalls in keiner Weise geändert und besteht in gleichem Grade bis zum heutigen Tage.

Im Laufe der Zeit wurden die Anfälle von Kopfschmerzen heftiger, waren von längerer Dauer und auch die Verdrehung des Auges nach dem Anfalle war eine hochgradigere, 14 Tage und noch länger dauernde. Erst im 12. Lebensjahre bemerkte das Mädchen, dass sich gegen das Ende des Anfalles nicht bloss das Auge verdrehe, sondern dass auch das obere Lid herabfalle. Nach dem Anfalle ging die Ptose in einigen Tagen zurück, während die Abweichung des Auges nur sehr langsam verschwand. Wenn sich der folgende Anfall etwas früher einstellte, befand sich das Auge gewöhnlich noch in der abweichenden Stellung.

Seit den letzten 5 Jahren sind die Kopfschmerzen sehr intensiv und dauern 2-5 Tage; die Kranke muss während des Anfalles liegen. Die ersten 2-3 Tage erbricht sie einige Male, obzwar sie fast nichts ist und trinkt, da sie gleichzeitig an einer vollständigen Appetitlosigkeit leidet. Während des Anfalles verlangt sie von ihrer Umgebung vollständige Ruhe; sie selbst spricht fast gar nichts, macht so, wie wenn sie schliefe, kann aber wegen der Schmerzen nicht einschlafen; erst gegen das Ende des. Anfalles, wenn die Schmerzen einigermassen nachgelassen haben, stellt sich ein wirklicher Schlaf ein, der dann längere Zeit dauert. Nach demselben ist, wenn die Kranke erwacht, der Anfall gewöhnlich beendet. Krämpfe oder überhaupt irgend welche andere Komplikationen der Augenmuskellähmung wurden weder von der Kranken selbst noch von deren Umgebung beobachtet. Die abweichende Stellung des Auges ist eine dauernde und besteht auch nach dem Anfalle in gleichem Grade fort. Nur die Ptose bessert sich allmählich, obzwar auch diese seit August vorigen Jahres nach einem besonders heftigen, 5 Tage dauernden Anfall von Kopfschmerzen nur unvollständig und sehr langsam verschwindet, so dass sich der neue Anfall früher einstellt, bevor noch die Ptose völlig verschwunden ist.

DiePeriode der Anfälle ist scheinbar eine unregelmässige; manchmal kommt der neue Anfall schon nach 14 Tagen, manchmal aber erst nach 3 Monaten; doch bleibt die Zahl der während eines Jahres sich einstellenden Anfälle im allgemeinen ungefähr die gleiche. Der Vater notiert seit 15 Jahren die Anfälle und konstatiert, dass die Zahl der jährlichen Anfälle zwischen 
12 und 17 schwankt. Im Sommer, wo die Patientin mehr in freier Luft arbeitet, ist die Zahl der Anfälle kleiner.

Die einzelnen Anfälle ähneln zwar einander vollkommen, was den Sitz und die Art der Kopfschmerzen, das Erbrechen, die Lähmung usw. anbelangt; aber ihre Intensität und Dauer schwanken ganz beträchtlich. Teilweise richtet sich die Intensität der Anfälle nach der Dauer des vorangehenden Intervalls, indem nach einer längeren Pause der erste Anfall gewöhnlich sehr heftig war and umgekehrt auf einen heftigen, lang dauernden Anfall gewöhnlich eine längere Pause folgte. Eine Regel stellte aber dieses Verhältnis keineswegs dar.

Die Kopfschmerzen sassen stets in der linken Kopfhälfte, und zwar schildert sie die Patientin als ein Bohren, das aus dem Innern des Schädels in das linke Auge und in die linke Schläfe (d. i. in die von der Lähmung ergriffene Seite) ausstrablte. Der Anfall begann abends oder am Morgen beim ersten Erwachen. Das Erbrechen war im Beginn des Anfalls reichlich, später blieb gewöhnlich nur Appetitlosigkeit und Unlust zurück. Eine Aura oder ein szintillierendes Skotom wurde nie beobachtet.

Die Menses bestanden seit dem 13. Lebensjahre, waren stets regelmässig und hingen mit den Anfällen in keiner Weise zusammen. Seit 3 Jahren bemerkt die Patientin eine Struma mässigen Grades, die aber auf den Verlauf der Krankheit keinen Einfluss besitzt.

Status praesens vom 24. November 1905 (14 Tage nach dem letzten Anfalle). Die Kranke ist entsprechend entwickelt und genährt. Der Kopf ist symmetrisch, im allgemeinen gut formiert; das Gesicht aber weist Differenzen anf. Die Augenbrauen stehen zwar auf beiden Seiten in gleicher Höhe, aber das Oberlid des linken Anges steht tiefer, so dass beim Blick nach vorn der Rand des Oberlides etwa $1 \mathrm{~mm}$ oberhalb der Hornhautmitte verläuft; bringen wir dagegen das gesunde Auge in eine analoge Position wie das linke, dann verläuft der obere Lidrand etwa $1 \mathrm{~mm}$ oberhalb des Hornhautrandes. Die linke Augenspalte misst etwa $1 / 2 \mathrm{~cm}$, die rechte dagegen etwa $1 \mathrm{~cm}$. Beim Blick nach oben wird das linke Oberlid um etwa $2 \mathrm{~mm}$ gehoben, während das rechte mit dem Bulbus so weit nach oben steigt, bis es ganz hinter dem Orbitalrand verschwindet. Der Lidschluss erfolgt beiderseits ganz normal und mit gleicher Kraft.

Bei ruhigem Blick nach vorn ist der linke Bulbus derart nach aussen gedreht, dass der Hornhautlimbus den äusseren Winkel berührt; dabei blickt der linke Bulbus zugleich etwas nach unten. Bei der Fixierung verfolgt der linke Bulbus den Finger nur nach aussen und zwar ebensoweit wie der rechte. Auch nach innen bewegt sich der linke Bulbus; aber diese Bewegung erfolgt relativ langsam und reicht nicht einmal bis zur Mittellinie. Nach oben fehlt die Beweglichkeit des linken Bulbus vollkommen. Nach unten bewegt sich derselbe nur ganz wenig, dreht sich aber dabei gleichzeitig in der Sagittalebene um etwa $3 \mathrm{~mm}$. normal.

Die Bewegungen des rechten Bulbus erfolgen nach allen Richtungen

Die linke Pupille ist stets etwas dilatiert, reagiert weder auf Licht noch auf Akkomodation, noch konsensuell. Die rechte Pupille reagiert auf Licht sehr prompt, desgleichen auch konsensuell und auf Akkomodation.

Die Augenmedien sind beiderseits klar; links besteht ein beträchtlicher Astigmatismus der Cornea. Ophthalmoskopisch weisen beide Augen keine 
Veränderungen auf. Papille und Netzhaut des linken Auges zeigen dieselbe Färbang und Form wie auf dem rechten Auge. Visus des rechten Auges nach Korrektion der Myopie normal, mit dem linken Auge zählt die Kranke Finger auf $5 \mathrm{~m}$, lesen kann sie aber mit demselben nicht einmal aus der Nähe.

Die Perimetrierung des rechten Auges ergibt einen normalen Befund, links lassen sich die Grenzen (die im grossen und ganzen von normaler Ausdehnung sind) mit Sicherheit nur für Blau bestimmen, schwieriger ist die Bestimmung derselben für Rot und ganz unmöglich für Grün. Diese beiden Farben, besonders aber die letztere, unterscheidet die Kranke mit dem linken Auge überhaupt schlecht; doch ist in dieser Hinsicht kein auffallender Unterschied zwischen dem Zentrum und der Peripherie des Gesichtsfeldes zu finden.

Ausser einer Struma mässigen Grades ist am ganzen Körper sonst keine Abnormität zu konstatieren. Geruch und Geschmack sind unverändert, ebenso das Gehör. Der N. trigeminus ist nicht schmerzhaft, die Sensibilität des Gesichtes ist auf beiden Seiten gleich. Reflexe normal;: Romberg negativ, in keiner Extremität Ataxie. Organe gesund.

\section{Decursus morbi.}

Am 8. XI. stellte sich der erste Anfall von Kopfschmerzen und Erbrechen ein; erst am nächsten Tage war eine vollkommene Ptose des linken Auges vorhanden.

10. XI. Der Kopfschmerz hat gänzlich aufgehört. 3 Tage später ging die Ptose auf den ursprünglichen Zustand zurück. Die Stellang und die Bewegungen des linken Auges blieben unverändert.

1. XII. Gestern Nachmittag stellte sich ein leichter Kopfschmerz ein, seit heute fruth aber besteht ein Anfall in voller Intensität. Die Patientin lokalisiert den Schmerz in die linke Stirngegend und ins linke Auge. Derselbe hat einen kontinuierlichen, zeitweise exazerbierenden Charakter. Zudem besteht seit gestern schon eine Magenverstimmung und noch gestern Abend erbrach die Kranke eine grünliche, mit Schleim gemischte Flüssigkeit, deren Menge ungefähr ein Liter betrug und die eine starke Reaktion auf Salzsäure gab. Der objektive Befund ist sowohl bezüglich der Ophthalmoplegie, als auch bezüglich der Empfindlichkeit im Bereiche des $\mathrm{N}$. trigeminus bis jetzt vollständig unverändert (wie vor dem Anfall). Auch der ophthalmoskopische Befund weist keine auffallenden Veränderungen auf, nur ist die Gefässpulsation noch deutlicher und greift auch auf die Venen über. Heute früh hat sich das Erbrechen wiederholt.

2. XII. Der Kopfschmerz hält an. Gestern ass die Kranke den ganzen Tag nichts, das Erbrechen aber hat aufgehört. Das linke obere Augenlid hängt mehr herab und verschliesst die Augenspalte fast vollständig. Die Bewegungen des linken Bulbus sind dieselben geblieben. Die Conjunctiva der Lider und des Bulbus des linken Auges ist hyperämisch. Sonst ist der Befund am übrigen Körper negativ. Der Visus beider Augen ist unverändert.

4. XII. Der Kopfschmerz hat nachgelassen. Das linke obere Augen- 
lid ist bis jetzt noch ad maximum herabgesunken und hebt sich nicht im geringsten beim Blick nach oben.

9. XII. Die linke Augenspalte hat sich wiederum geöffnet, die Ptose nimmt ab.

16. XII. Gestern Nachmittag begann ein neuer Anfall, aber das linke obere Augenlid verharrt in demselben Zustand wie im Zustand der Euphorie. Die Bindehaut der Lider und des Bulbus ist wiederum injiziert.

17. XII. Der Schmerz und das Erbrechen bestehen fort. Es hat sich wiederum eine vollständige Ptose eingestellt.

18. XII. Der Schmerz hat aufgehört.

19. I.-24. I. 06. Ein neuer Anfall, nach welchem die Ptose 14 Tage anhielt.

17. II. Gestern Abend begann ein neuer Anfall. Das linke obere Augenlid ist leicht ödematös, seine Venen schimmern durch die Haut besser hindurch und sind im Vergleich zu den rechtsseitigen offenbar erweitert. Auch die Skleralvenen sind gegenüber jenen der gesunden Seite deutlich voller. Bei Kompression der linken Carotis (einwärts vom M. sternocleidomastoideus) mit dem Daumen schwellen binnen kurzem alle diese Venen des Lides und der Sklera wesentlich ab und werden blass. Gleichzeitig lässt der Kopfschmerz für diesen Moment nach. Nach Phenacetin $(0,5 \mathrm{~g})$ hörte der Kopfschmerz am Nachmittag auf. Die Ptose des linken Auges hat nicht zugenommen.

24. II. Seit den letzten 3 Tagen bestehen wiederum Kopfschmerzen. Der linke Bulbus zeigt wiederum eine Hyperämie der Venen and eine mässige Protrusion. Totale Ptosis. M. trochlearis und abducens des kranken Auges funktionieren gut.

12. III. Gestern begann gegen Morgen ein neuer Anfall. Hente Vormittag bekam die Kranke $0,5 \mathrm{~g}$ Phenacetin, worauf der Schmerz am Nachmittag aufhörte.

13. III. Der Kopfschmerz hat sich wiederum eingestellt, aber in geringerem Grade. Die Ptose des linken Auges hat unbedeutend zugenommen.

29. III. Gestern Nachmittag stellte sich ein neuer heftiger Anfall ein. Im Laufe des Tages bekam die Kranke zweimal je $1 / 2$ g Phenacetin.

30. III. Der Kopfschmerz dauert fort. $1 / 2 \mathrm{~g}$ Phenacetin.

31. III. Die Kranke bekam wiederum ein Phenacetinpulver, aber der Kopfschmerz hörte erst am Nachmittag auf. Die Ptose ist bereits seit gestern eine rollkommene.

Bis zum 10. IV. 1906, an welchem Tage die Kranke entlassen warde, stellte sich kein neuer Anfall ein. Sie wurde ungeheilt entlassen. Sie bekam während der ersten 2 Monate Jodkali ( $1,5 \mathrm{~g}$ pro die), während der folgenden 2 Monate Bromkali ( $3 \mathrm{~g}$ pro die) und während der letzten Zeit nur Phenacetin im Anfall. Im Anfang wurde anch die Galvanisation des Kopfes und des Sympathicus versucht.

Aus dem Obigen geht hervor, dass es sich um einen typischen Fall von ophthalmoplegischer Migräne handelte. Die Lähmung ist bier, wie es in reinen Fällen verlangt wird, nur auf den N. oculomotorius 
der einen Seite (der linken) lokalisiert und ist eine totale. Die von diesem Nerven versorgte Bulbusmuskulatur war zur Zeit der Beobachtung bereits dauernd gelähmt (M. rectus sup., internus und inferior und die Inneńmuskulatur des Auges); eine periodische Exazerbation der Lähmung betraf nur den M. levator palpebrae superioris, der in den freien Intervallen nur eine geringe Parese darbot.

Da die Kranke an der periodischen Oculomotoriuslähmung bereits etwa 15 Jahre leidet und jährlich durchschnittlich etwa 15 Anfälle durchmacht, so hat sie im ganzen mehr als 200 Anfälle überstanden und zwar anfangs nur Paresen, später vollständige Lähmungen des linken Oculomotorius. Unter diesen Verhältnissen kann man wohl schwerlich annehmen, dass irgend ein rezidivierender organischer Prozess die einzige und ausschliessliche Ursache der beschriebenen Anfälle sei. Schon der Kopfschmerz hat in unserem Fall einen so deutlich ausgeprägten migränösen Charakter, dass er sich mit der gewöhnlichen Cephalalgie nicht vergleichen lässt.

Allein es lässt sich schwer entscheiden, ob es sich um eine idiopathische (echte) oder eine symptomatische Migräne handelt. Diese Frage ist der Gegenstand eines langen und bis jetzt noch nicht entschiedenen Streites zwischen den Anhängern Charcots einerseits und jenen Möbius' andererseits. Die Unmöglichkeit einer definitiven Entscheidung in diesem Streite hat darin ibren Grund, dass wir das Wesen der Migräne überhaupt nicht sicher kennen und keine differentiellen Kriterien zwischen der idiopathischen und symptomatischen Migräne besitzen.

Karplus ${ }^{5}$ ) sagt, es müsse zwischen symptomatischer und idiopathischer Migräne, was das klinische Bild betrifft, kein Unterschied sein; ob aber diesen beiden Formen der Migräne derselbe Hirnprozess zugrunde liege, wolle er nicht entscheiden.

Möbius hält zwar die die periodische Oculomotoriuslähmung begleitende Migräne für symptomatisch, erklärt aber ihren Anfall für einen echten Migräneanfall*) und nicht für eine einfache Cephalalgie, die eine nur scheinbare Ähnlichkeit mit einem Migräneanfall besitzt.

*) Möbius sagt wörtlich: . . „, ,in denen (i. e. in den Fällen von sym. ptomatischer Migräne) echte Migräneanfälle a ș Symptom nicht der Krankheit Migräne, sondern anderer Gehirnkrankheiten auftreten." (Die Migräne. Nothnagels spezielle Pathol. u. Therapie. 1894. Bd. 12. Heft 2. S. 72.)

An einer anderen Stelle: „Nach meiner Auffassung ist die Migräne bei der periodischen Oculomotoriuslähmung eine symptomatische Migräne, wie sie bei verschiedenen Gehirnkrankheiten (Epilepsie, progressiver Paralyse, arteriosklerotischen Herden) vorkommt." (Deutsche Zeitschrift für Nervenheilkunde. 1900. Bd. 17. S. 301.) 
Für die symptomatische und gegen die idiopathische Migräne führt Möbius ${ }^{13}$ ) folgende Gründe an:

Die periodische Oculomotoriuslähmung soll zum Unterschied von der idiopathischen Migräne manchmal einen atypischen Verlauf haben. Der Schmerzanfall dauert in solchen atypischen Fällen angeblich 3-4 Wochen, ja sogar einige Monate und dann erst stellt sich die Lähmung ein; ferner sollen auch die freien Intervalle manchmal abnorm lange, eventuell auch mehrere Jahre dauern. Gegenüber dieser Einwendung ist aber zu erwägen, dass gerade diese Fälle, die sich durch lange und ungleiche Intervalle sowie durch mehrere Wochen dauernde Schmerzen auszeichnen, strittig sind, und dass man in denselben eher eine einfache rezidivierende Lähmung auf organischer Basis, eventuell in Kombination mit echter Migräne annehmen kann. Selbst Möbius ist in der letzten Zeit bei der Klassifikation der publizierten Fälle sehr vorsichtig und scheidet die Mehrzahl derselben aus der periodischen Oculomotoriuslähmung aus; jene Fälle, die er gelten lässt, zeigen einen ziemlich periodischen, der typischen Migräne sehr ähnlichen Verlauf. Was in unserem Falle und auch bei den übrigen typischen Fällen im Gegensatz zu der echten Migräne auffällt, ist nur die etwas längere Dauer des Schmerzanfalls. Dieser Unterschied ist aber nicht so gross, wie Möbius ihn hinstellt. Am häufigsten dauert der Anfall der ophthalmoplegischen Migräne 2-3 selten 5-8 Tage; jene Fälle, in denen der Kopfschmerz länger als eine Woche dauert, gehören zu den Ausnahmen und sind auch noch in anderer Beziehung wenig typisch. Übrigens tritt auch die idiopathische Migräne manchmal in Form des Status migraenosus auf, der viele Tage und vielleicht auch mehrere Wochen dauern kann.

Ferner führt Möbius an, dass die die periodische Oculomotoriuslähmung begleitende Migräne stets auf dieselbe Seite beschränkt bleibt, während die idiopatbische Migräne sehr häufig abwechselnd beide Seiten befällt. Dem ist wiederum hinzuzufügen, dass auch nach der Statistik Möbius jene Fälle, in denen auch die idiopathische Migräne stets auf dieselbe Kopfhälfte beschränkt bleibt, nicht gerade selten sind. Unter 82 Fällen von idiopathischer Migräne gibt Möbius 17 an, in denen die Migräne "stets oder fast stets" auf der rechten, und 23 Fälle, in denen dieselbe „stets oder fast stets" auf der linken Seite sass; also fast in 50 Proz. der Fälle ist auch die idiopathische Migräne einseitig.

Den wichtigsten Beweis gegen die idiopathische Natur der Migräne erblickt Möbius in dem Mangel der Heredität in der Anamnese der mit periodischer Oculomotoriuslähmung behafteten Patienten. Wenn wir aber alle bekannt gewordenen, namentlich aber die in der jüngsten Zeit publizierten Fälle von ophthalmoplegischer Migräne nach dieser Hinsicht prüfen, so finden wir, dass dieser Beweis nicht für alle Fälle 
Geltung besitzt In unserem Falle sowle im exsten Falle Rybas') vermıssen wır zwar die Hereditat der Migrane, dagegen fubrt Le c] éz $10^{\text {} \nsim}$ ) zum Schutze der Charcotschen Theorie folgende Falle an, in denen die Ascendenten an Migrane litten Bernbaridt ${ }^{14}$ ), de Lapeisonne, Joachım ${ }^{15}$, Lafon und Willemonte, Karplus, ausserdem litten Im Falle Chabberts em Bruder und im Falle Serffers 3 Kunder des Kranken an gewohnlicher Migrave $\mathrm{Ob}$ es sich in allen diesen Fallen um eine ophthalmoplegische Migrane gehandelt hat, will ich nicht entscheiden, doch sind einige ron diesen Fallen sehr typisch

Den Mangel einer Aura uberhaupt und speziell einer visuellen halt Mobıus ebenfalls fur einen entscheidenden Beweis gegen dieıdiopathische Natur der Migrane Aber nicht einmal in dieser Hinsicht sind die als ophthalmoplegische Migrane (oder periodische Oculomotoriuslahmung) geführten Falle ohne Ausnahmen De Lapersonne, Selffer, Karplus fuhren, abgesehen von dem strittigen Falle Chabberts, eme leichte visuelle Aura vor dem Anfall an Ferner muss man bedenken, dass eme visuelle Aura kein unanfechtbaier Beweıs fur die ıdıopathische Migrane ist Zwar gibt Gowers ${ }^{16}$ ) an, dass die Halfte aller Falle von gewohnlicher Migrane eine visuelle Aura besitze, aber Mobius bezeichnet diese Angabe als sicher zu hoch gegriffen und gibt an, dass er eine visuelle Aura nur in etwa 10 Prozent der Falle von Migrane gefunden habe Es ist aber fraglich, ob auch diese Berechnung Mobius der Wrrklichkelt entspiıcht, denn die ophthalmische Migrane kann nach dem ubereinstımmenden Urteil vielleicht aller Neurologen (Mobiu s nicht ausgenommen) auch ein Symptom anderer Krankheiten sein, wie Tabes progressiver Paralyse, Tumors oder Lues des Gehinns, speziell aben der Epilepsie Krafft-Ebing ${ }^{17}$ ) und viele andere Autoren, unter ihnen anch Mobius, behaupten, dass die symptomatische Migrane gewohnlich eine ophthalmische ser, so dass der Mangel einer visuellen Auıa bei den Anfallen der periodischen Oculomotoniuslahmung eher gegen die symptomatische Migrane als gegen die idıopathsche zeugt

Trotz dieser Einwendungen will 1ch jene Grunde, aus denen sich Mobius bel der periodisehen Oculomotoriuslahmung fur die symptomatische Migiane entschieden hat, nucht unterschatzen Die Bedeutung derselben lasst sich nicht vollstandig in Abrede stellen, mag auch da und dort eine Einwendung moglich sein Namenthich ist es die Einseltigkeit der Kopfschmerzen und der Lahmung, die uns zwingt, bestimmte Unterschiede zwischen der ophthalmoplegischen und der ge-

*) Der zwente Fall Rybas weist zwar Hereditat auf, doch ist der Fall selbst nicht typisch

**) Die neueren Falle beschreibt Leclézio selbst in extenso 
wohnlichen Migrane anzunehmen, die man nicht so leicht ubergehen kann, wie dies die franzosischen Autoren tun

Seiffe 1, Spitzer und in letzter Zeit auch Karplus trachten diesen Unterschied zwischen der deutschen und franzosischen Schule dadurch auszugleıchen, dass sie die als periodische Oculomotoriuslahmung oder als ophthalmoplegische Migrane beschriebenen Falle in zwel Gruppen teilen In jenen Fallen, wo sich die Erblichkeit der Migrane nachweisen lasst und wo eventuell auch Anfalle von einfacher Migrane (ohne Lahmung) den Anfallen der ophthalmoplegischen Migrane jahielang vorangehen oder mut ihnen abwechseln, erkennen diese Autoren die idropathische Migrane an In den ubrigen Fallen wuide es sich sodann um eine symptomatische Migrane hardeln Die Giunde Mobıus' gegen die 1diopathische Migrane (Einsertigkeit usw) trachten diese Autoren dadurch zu umgehen, dass sie in den betreffenden Fallen eine gewisse (intracranielle) lokale Disposition annehmen, die nach Spitzeis Erklarung eine angeborene oder erworbene sein konnte und die nie an und fur sıch, sondern erst in Kombination mit einem Migıaneanfall eine Oculomotoriuslahmung hervorrufen konnte

Diese Disposition musste aber nicht allein die Lahmung, sondern auch die Migrane selbst betreffen, damit wil uns nicht nul die standige Einseitigkeit der Lahmung, sondern auch die standige Einseitıgkeit der Migrane, wie es eben in den Fallen von ophthalmoplegischer Migrane typisch 1st, erklaren konnen

Im ubıigen hat die Annahme eıner personlichen Disposition zur ophthalmoplegischen Migrane vieles fur sich, da sie sehr wahrscheinlich ist Auf diese Weise konnten wil den Einwand Mingazzinıs, dass die ophthalmoplegische Migrane oft schon in einem sehr zarten Alter beginne*), sowle auch den Einwand von Mobius, dass eine Oculomotoriusldhmung selbst in schweren Fallen von idiopathischei Migrane nicht vorkomme, spielend erklaren

Wenn wir aber auch zugeben, dass die die periodische Oculomotoriuslahmung begleitende Migrane nicht nur eine idiopathische, sondein auch eine symptomatische sein kann, so ist damit die Streitfiage zwischen den Ansichten von Charcot und Mobius nicht vollstandig gelost Denn der Kern dieser Streitfrage beruht nicht darn, ob sich hinter der ophthalmoplegischen Migrane eine Idiopathische oder symptomatische

*) Im ersten Lebensjahre beobachteten die Entstehung der ophthalmoplegischen Migrane Mobius, Karplus, Ormerod und Spitzer, Im zweiten Lebensjahre Snell, de Schweinitz Im Falle Russels trat der erste Anfall der Migrane 14 Tage nach der Geburt auf, die Lahmung gesellte sich erst Im 6 Lebensjahre hunzu 
Migräne verbirgt, sondern darin, ob, was den Kausalnexus betrifft, die Migräne primär und die Lähmung eine Folge derselben ist.

Wenn wir in einem bestimmten Falle die Migräne als idiopathisch bezeichnen, so erklären wir gewiss schon hierdurch die Lähmung als eine sekundäre, durch die Migräne hervorgerufene Erscheinung. Dieses Urteil lässt sich aber bei der symptomatischen Migräne nicht umkehren, da auch die symptomatische Migräne, wenn es sich bei dieser ebenfalls um einen echten Migräneanfall handelt (siehe Seite 192), gewiss ebensogut eine Oculomotoriuslähmung hervorrufen kann wie die idiopathische Migräne, denn dann sind die Anfälle der symptomatischen und der idiopathischen Migräne in der Handlung eigentlich identisch und unterscheiden sich von einander nur durch den Ursprung, resp. die Veranlassung. Mag auch daher die Migräne vielleicht durch irgend eine Läsion hervorgerufen sein, so kann dennoch die Lähmung eine Folge der Migräne und muss nicht die unmittelbare Folge jener Läsion sein, die die Migräne hervorgerufen hat.

Mit dieser Ansicht stehe ich keineswegs allein da. Aus einem wörtlichen Zitat bei Karplus ersehe ich, dass sich sçhon Spitzer von den übrigen Autoren getrennt hat und dass er, ausgehend von der Ansicht, dass bezüglich Qualität und Handlung des Anfalls die Migräne stets die gleiche ist, behauptet, dass nicht nur die idiopathische, sondern auch die symptomatische Migräne eine periodische Oculomotoriuslähmung hervorrufen kann. In Fällen von symptomatischer Migräne nimmt er an, dass erentuell dieselbe Grundkrankheit, welche diese Migräne hervorruft, auch die erwähnte Disposition zur Ocumolotoriuslähmung bedingt, denn er hält es für ausgeschlossen, dass ein Anfall von, sei es idiopathischer oder symptomatischer, Migräne, als solcher allein, so schwere Symptome wie eine vollkommene Oculomotoriuslähmung hervorrufen könnte.

Ich glaube, dass auch die französische Schule das Hauptgewicht nicht auf den. Umstand legt, dass es sich bei der ophthalmoplegischen Migräne um eine idiopathische Migräne handelt. Wie aus der Abhandlung Leclézios (u. a.) hervorgeht, bestreben sich die französischen Autoren in einem gegebenen Falle nur zu entscheiden, ob die Hemicranie, die der Oculomotoriuslähmung vorangeht, eine echte Migräne ist (migraine vraie) oder eine falsche (migraine fausse, céphalée banale). Auch Chareot selbst sowie Ballet, de Lapersonne u. a. sprechen nirgends von einer idiopathischen Migräne, sondern nur von einer echten Migräne, indem sie analog wie $G$ owers und viele andere Autoren annehmen, die Migräne könne zwar eine verschiedene Ätiologie haben, aber was das Wesen und die Handlung des Anfalls anbelangt, gebe es nur eine einzige Form derselben, nämlich die echte. $\mathrm{Ob}$ die 
ophthalmoplegische Migräne eine angeborene oder eine erworbene ist, darüber sprechen sie sich nicht aus; aber aus dem ganzen Inhalt ihrer Abhandlungen geht hervor, dass sie beide Möglichkeiten zulassen.

Suchen wir nun in den Schriften von Möbius nach den Gründen, aus denen er die Migräne (auch die symptomatische) nicht als primär, d. i. als Ursache der Lähmung gelten lässt, so finden wir hauptsächlich folgende zwei Gründe:

Zunächst weist Möbius darauf hin, dass die Kopfschmerzen in der Regel aufhören, sobald die Lähmung eingetreten ist, was ein Beweis dafür sein soll, dass die anf die Lähmung abzielenden Läsionen die Migränesymptome hervorrufen und nicht diese jene. Diese Ansicht scheint mir nicht hinreichend begründet zu sein, weil man ja aus denselben Gründen sagen könnte: Die Migräne hört auf, sobald sie die Lähmung hervorgerufen hat. Übrigens entsteht, wie unser Fall sowie auch der Fall Rybas und, wie ich glaube, die Mebrzahl der neueren typischen Fälle*) beweist, die Paralyse oft noch während der Schmerzen, ja Seiffer, de Lapersonne, Russel, Paderstein bemerken ausdrücklich, dass sich die Oculomotoriusstörung sofort wieder besserte, sobald die Schmerzen aufhörten.

Einen zweiten Grund gegen die primäre Migräne erbliekt Möbius in dem Umstande, dass sich die idiopathische Migräne sehr selten mit der Oculomotoriuslähmung kombiniert, obwohl man nach diesem Prinzip erwarten sollte, dass sich die Lähmung wenigstens nach einem jeden schweren Migräneanfall zeigen werde. Dagegen lehre die Erfahrung, dass die Migräne höchstens erst nach vieljähriger Dauer im vorgeschrittenen Alter zu gröberen Störungen führe, während die periodische Oculomotoriuslähmung gewöhnlich im zarten oder wenigstens im jugendlichen Alter beginne und gleich im ersten Anfalle nicht nur die Migräne, sondern auch die Lähmung, vorhanden sei.

Dieser zweite Grund ist an und für sich ziemlich äberzeugend; sobald wir aber die individuelle lokale Disposition berücksichtigen, welche Seiffer und Karplus bei der idiopathischen und Spitzer auch bei der symptomatischen Migräne annehmen (Seite 195), entfällt auch dieser zweite, scheinbar gegen die Migräne als primäre Erscheinung sprechende Grund.

Andererseits können wir dagegen mehrere Gründe anführen, die für eine primäre Migräne und sekundäre Lähmung sprechen:

Zunächst ist es die konstante Erscheinung, dass der Anfall der periodischen Oculomotoriuslähmung mit der Migräne beginnt und erst

*) Unter anderen gehören alle 3 Fälle Karplus' hierher. 
wenn diese 2-3 Tage gedauert hat, die Oculomotoriusparese eintritt, die am 4. und 5. Tag in eine komplete Lähmung übergeht, wenn der Kopfschmerz, resp. die Migräne nicht aufhört. In leichteren Fällen bessert sich die Lähmung, sobald der Schmerz aufgehört hat. So verhielt sich die Lähmung in unserem und Rybas Falle und in der Mehrzahl der typischen Fälle überhaupt (Vissering, Senator ${ }^{18}$ ), Seiffer, Karplus, Paderstein ${ }^{19}$ ), Russel, de Lapersonne u. a. m.). In leichten Anfällen, in denen die Migräne nur 1-2 Tage dauert, stellen sich seitens des Oculomotorius überhaupt keine Symptome ein oder dieselben sind nur angedeutet $\left(\mathrm{Manz}^{20}\right)$, Senator, Suckhing, Kollarits, Darkschewitsch u. a.) Jene Fälle, in denen der Schmerz fehlte oder die Lähmung dem Schmerz voranging, gehören nicht in das Gebiet der ophthalmoplegischen Migräne.

Der Verlauf der ophthalmoplegischen Migräne stimmt mit dem Verlauf der gewöhnlichen Migräne vollkommen überein. Es ist bekannt, dass die Anfälle der gewöhnlichen Migräne von der Jugend gegen das Mannesalter an Stärke zunehmen. Analog beobachtet man auch bei der ophthalmoplegischen Migräne im zarten oder jugendlichen Alter mehrere Jahre hindurch eine einfache Migräne ( $\mathrm{Manz}{ }^{21)}$ Borthen, Ballet ${ }^{22}$ ), Russel, Seiffer, unser Fall u. a.). Mehrere Autoren (Russel, Seiffer u. a.) geben an, und auch ich habe in unserem Falle beobachtet, dass der Kopfschmerz und das Erbrechen sowie die nachfolgende Lähmung von um so grösserer Intensität und längerer Dauer waren, je länger das freie Intervall war, das voranging. In analoger Weise verhält sich auch die gewöhnliche Migräne.

Es ist ferner bekannt, dass durch eine zeitweilige Veränderung des Klimas oder durch die Gravidität die Migräneanfälle gemildert werden, und in ähnlicher Weise wurde schon öfters beobachtet, dass die ophthalmoplegische Migräne in der Schwangerschaft entweder ganz und gar zum Stillstand kam (zwei Fälle von Karplus) oder in eine gewöhnliche Migräne ohne Lähmung mit leichteren Anfällen überging (Fall Senators). Der Vater unserer Patientin gab an, dass dieselbe im Sommer, wo sie mehr im Freien arbeitete, nicht so viele Anfälle hatte wie im Winter.

Ich machte ferner die Beobachtung, dass man durch rechtzeitige Verabreichung von Phenacetin nicht bloss den Kopfschmerz unterdrücken, sondern bei manchen Anfällen sogar die Lähmung verhüten konnte, so dass sie sich überhaupt nicht zeigte. Diesen Einfluss des Phenacetins auf den Kopfschmerz und die Oculomotoriuslähmung verzeichnen auch Ryba und Karplus. Auch Bernhardt ${ }^{14}$ ) und Vissering konstatierten den segensreichen Einfluss des Antipyrins auf die Anfälle der ophthalmoplegischen Migräne. Wäre die Lähmung nicht eine 
Folge der Migräne, dann könnten zwar nach dem Phenacetin die Kopfschmerzen aufhören, aber die Lähmung müsste troțdem in entsprechendem Grade auftreten.

Bei den gewöhnlichen basalen Prozessen (lokalen Meningitiden, Tumoren) überwiegt im klinischen Bilde mehr die Lähmung der Gehirnnerven als der Kopfschmerz, ja oft ist die Lähmung das einzige Symptom. Die motorischen Nerven unterliegen dem Druck sebr leicht*); es müsste daher die Lähmung oft gleichzeitig mit dem Kopfschmerz eintreten, wenn eine Lässion bei der periodischen Oculomotoriuslähmung die unmittelbare Ursache der Lähmung wäre.

Dass die Grundlage der ophthalmoplegischen Migräne die echte Migräne ist, erschliesse ich auch aus dem Unterschied in der Statistik dieser Krankheit in Bezug auf das Geschlecht. Schon Senator fiel es auf, dass die periodische Oculomotoriuslähmung häufiger bei Franen vorkommt als bei Männern. Die neueren Fälle, zu denen auch der Fall Rybas und der unserige gehören, sind weitere Beweise hiefür. Aus einer Vergleichang aller typischen Fâlle von ophthalmoplegischer Migräne, soweit mir dieselben, wenigstens im Referat, zugänglich waren, ziehe ich den Schluss, dass 60-70 Proz. derselben auf Frauen entfallen. Ein ähnliches Verhältnis geben die Autoren auch bei der gewöhnlichen Migräne an.

Aus allen diesen Gründen geht einerseits hervor, dass die ophthalmoplegische Migräne einen ganz ähnlichen Verlauf hat wie die gewöhnliche Migräne, andererseits dass sich Oculomotoriuslähmung nach dem Kopfschmerz richtet und nicht umgekehrt. Ich halte es daher für ausgeschlossen, dass die Migräne und Lähmung nur einfache Folgezustände einer exazerbierenden basalen Läsion oder einer zufälligen Hyperämie sein sollten, wie Karplus meint. Damit entfällt auch die ursprüngliche Ansicht von Möbius, der eine einfache Läsion im Pedunculus nahe dem Oculomotoriuskern annahm.

Anders verhält sich aber die Sache mit jener Theorie, welche Möbius in der letzten Zeit zur Erklärung der periodischen Oculomotoriuslähmung aufgestellt hat. Zur Widerlegung dieser Theorie reichen Gründe, welche beweisen, dass die Ursache der Oculomotoriuslähmung: eine echte Migräne sei, nicht ans, weil Möbius nicht mehr behauptet, dass die Läsion selbst die unmittelbare Ursache dieser Lähmung ist, sondern der Anfall von Hyperämie in der Umgebung des betreffenden Oculomotorius an der Gehirnbasis. Statt des Migräneanfalls supponiert er hier als Ursache der Lähmung einen Anfall, der zwar

*) Bei der gewöhnlichen Radialislähmung bleibt die Funktion der sensitiven Nerven ungestört. 
qualitativ verschieden, aber durch sein ganzes Wesen und namentlich durch seine Entstehung - wie Möbius selbst zugibt - dem echten Migräneanfall sehr ähnlich ist. Möbius sagt ausdrücklich, das für die Erklärung der Periodizität bei der periodischen Oculomotoriuslähmung am besten die Erklärung nach Art einer Explosion passe, die er auch bei der Epilepsie und der Migräne für die wahrscheinlichste halte. Eine angeborene Läsion, i. e. ein benigner Tumor des Oculomotorius, sei nur die Grundursache der Krankheit, der die Rolle einer dauernden Reizung zukomme, infolge deren sich ein Explosionsstoff ansammle. Bei einer bestimmten Gelegenheit (also wie bei der Migräne) komme es zur Explosion, auf welche der Organismus durch die bereits erwähnte Hyperämie in der Umgebung jener Läsion reagiere und als deren weitere Folgen die Oculomotoriuslähmung, der Kopfschmerz und das Erbrechen anzusehen sind. Die beiden letzten Symptome sollen die Folgen der Reizung der harten Hirnhaut sein. ${ }^{*}$ )

Unter solchen Umständen ist es wirklich schwer zu sagen, wodurch. sich ein Anfall der ophthalmoplegisehen Migräne von einem Anfall der gewöhnlichen Migräne unterscheidet. Schon früher stellte Möbius. die die periodische Oculomotorinslähmung begleitende Migräne. was die Qualität der einzelnen Anfälle anbelangt - als eine echte (wenn auch symptomatische) Migräne hin und nunmehr behauptet er überdies, dass auch das Wesen und die Entstehung des Anfalls der ophthalmoplegischen Migräne analog seien jenem bei der gewöhnlichen Migräne.

Nach Möbius beruht der Unterschied zwischen den Anfällen der ophthalmoplegischen und der gewöhnlichen Migräne darin, dass sich bei der ophthalmoplegischen Migräne der Anfall bloss durch eine lokale Reaktion äussert, während sich bei der idiopathischen Migräne die Hauptreaktion in der Gehirnrinde abspielen soll. Diese Differenzen besitzen aber keine entscheidende Bedeutung, da das Wesen der Migräne bis jetzt abberhaupt noch strittig ist und speziell noch nicht mit absoluter Sicherheit nachgewiesen wurde, dass die idiopathische Migräne, selbst wenn wir eine leichte, einfache Gesichtsaura gelten lassen, ihren Sitz im Grosshirn bätte (Seite 221 u. f.).

Jedenfalls nähert sich Möbius durch diese neue Erklärung der Charcotschen Auffassung der ophthalmoplegischen Migräne, denn er supponiert als unmittelbare Ursache der Oculomotoriuslähmung nicht mehr die Läsion, sondern den "Anfall", der dem Anfall bei der gewöhnlichen Migräne sehr ähnlich ist.

*) Auch bei der idiopathischen Migräne erklärt Möbius den Kopfschmerz und das Erbrechen als Symptome einer einseitigen Hyperämie der Gehirnhäute, speziell der Dura mater. 
Der einzige wesentliche Unterschied zwischen den beiden Ansichten beruht in der kausalen Erklärung der Lähmung. Möbius bestreitet bisher, dass ein Anfall der, wenn auch symptomatischen, Migräne die Ursache der Lähmung sein könnte, und ist mit Karplus der Meinung, dass der die periodische Oculomotoriuslähmung bedingende Anfall nur in einer lokalen Hyperämie in der Umgebung jenes benignen Tumors des $\mathrm{N}$. oculomotorius, resp. in der umgebenden Dura mater bestehe, und dass erst hierdurch zwei weitere, von einander unabhängige Symptome hervorgerufen werden: die Migräne und die Lähmung. Wäre aber Möbius bei der Erklärung dieser symptomatischen Migräne konsequent geblieben und hätte er denselben Weg eingeschlagen wie bei der Erklärung der Kopfschmerzen und des Erbrechens bei der idiopathischen Migräne, so hätte er die lokale Hyperämie der Dura mater selbst als Grundlage dieser symptomatisehen Migräne bezeichnen müssen und nicht als einen selbständigen Prozess, dem sich erst durch einen Kausalnezus die Migräne als ein zweiter selbständiger Prozess anschliesst. Analog der Möbiusschen ErkJärung der idiopathischen Migräne müssen wir die lokale Hyperämie der Dura, den Kopfschmerz und das Erbrechen als einen einheitlichen klinischen Vorgang, in diesem Falle nach Möbius als symptomatische Migräne auffassen. Der zweite selbständige, kausal zusammenhängende Prozess ist nur die Lähmung, die nach der eigenen Auffassung von Möbius hier nur aus dem Grunde auftritt, weil der N. oculomotorius mit einer angeborenen Läsion behaftet ist und daher einen Locus minoris resistentiae darstellt. Durch diese kleine, aber bedeutungsvolle, nach der eigenen Möbiusschen Erklärung der idiopathischen Migräne vorgenommenen Änderung in der kausalen Erklärung der einzelnen Erscheinungen der ophthalmoplegischen|Migräne lässt sich die Möbius sche Theorie mit jener Charcots leicht in Einklang bringen, wenn auch die Möbiussche Theorie sonst in den Einzelheiten unverändert bliebe.

Wenn ich mich nun auf Grundlage aller bis jetzt angeführten Urteile über die Ursache der periodischen Oculomotoriuslähmung aussprechen soll, muss ich als einzig wahrscheinliche Erklärung die Lehre Charcots hinstellen, rä̈mlich die, dass die echte Migräne die Ursache der periodischen Oculomotoriuslähmung sei. Ich will nicht behaupten, dass es sich stets um eine idiopathische Migräne handelt, sondern rechne auch die symptomatische Migräne hierher.

Wie und wo wir uns diesen lähmenden Einfluss der Migräne vorzustellen haben, ist eine zweite strittige Frage bei der ophthalmoplegischen Migräne, die daher besondẹrs besprochen werden muss. Diese Frage deckt sich zum Teil mit der Frage nach dem Wesen der 
Migräne überhaupt, denn ist die ophthalmoplegische Migräne wirklich eine echte Migräne, dann muss auch die die ophthalmoplegische Migräne bedingende Alteration eine grosse Ähnlichkeit mit jener Alteration haben, die die gewöhnliche Migräne hervorruft, oder gar mit ihr identisch sein.

Nach diesem Prinzip sollte man sicherlich zuerst eine Erklärung der Migräne überhaupt begründen und dann erst zur Erklärung der periodischen Oculomotoriuslähmung übergehen. Wenn wir uns aber mehr auf einer reellen Basis bewegen wollen, ist es besser, die Argumentation umzukehren und zuerst eine Erklärung der periodischen Oculomotoriuslähmung zu suchen, weil wir bei dieser Krankheit eher einen objektiven Befund haben als bei der gewöhnlichen Migräne, von der allgemein behauptet wird, dass sie keinen anatomischen Befund aufweise.

Es könnte vielleicht scheinen, dass, wenn wir das Charcotsche Prinzip, die ophthalmoplegische Migräne sei eine echte Migräne, wir damit auch seine Erklärung der Entstehung der periodischen Lähmung angenommen haben. Diese Folgerung ist aber nicht notwendig. Bis jetzt ist es noch nicht mit absoluter Sicherheit gelungen, eine Basis für die gewöhnliche Migräne zu finden ${ }^{23}$ ), und daher können wir, bevor ein Weg zur Erklärung der gewöhnlichen Migräne gefunden ist, auch eine neue Erklärung für den Kausalnexus zwischen Migräne und Oculomotoriuslähmung suchen, ohne von dem Charcotschen Prinzip abzuweichen. Spitzer z. B. erklärt das Wesen der Migräne in einer von allen anderen Autoren vollständig abweichenden Weise - durch Verschliessung des schon von vornherein relativ stenosierten Foramen Monroi und konsekutive Schwellung der betreffenden Hemisphäre - und doch ist auch er gleich Charcot der Ansicht, dass bei der periodischen Oculomotoriuslähmung der Migräneanfall die unmittelbare Ursache der Lähmung sei.

Es existiert eine ganze Reihe von Theorien über die Entstehung der Lähmung bei der ophthalmoplegischen Migräne, resp. bei der periodischen Oculomotoriuslähmung. Diese Theorien zerfallen in Bezug auf die grundsätzliche Auffassung dieser Krankheit in zwei Gruppen, je nachdem, ob der Autor die Krankheit nur als eine modifizierte, sonst aber echte Migräne auffasst, oder ob er den Migränecharakter leugnet. Die Theorie von Möbius, die ich eingehend erwähnt habe, steht an der Grenze dieser beiden Auffassungen, denn sie hält die Läsion für die entferntere und den Anfall für, die unmittelbare Ursache der Lähmung.

Da es sich nur um die Erklärung der periodischen Oculomotoriuslähmung mit Hilfe der echten Migräne handelt, entfallen für uns alle jene Theorien, die auf einer anderen Basis aufgebaut sind, weshalb ich auf dieselben nicht näher eingehen werde. Aus denselben Gründen 
kann ich die Theorie von Möbius nur mit jener Modifikation in Betracht ziehen, die ich oben begründet habe und welche dahin lautet, dass auch nach dieser Theorie der ophthalmoplegischen Migräne die Migräne, und sei es auch nur die symptomatische, die Ursache der Oculomotoriuslähmung sei.

Mit Rücksicht auf das oben Gesagte ist es klar, das uns in erster Linie die Charcotsche Erklärung der ophthalmoplegischen Migräne interessieren wird. Auf Grund der mir zugänglichen Literatur muss ich aber annehmen, dass sich Charcot über die Entstehung der Oculomotoriuslähmung in einem zweifachen Sinne ausgesprochen hat, obwohl er in beiden Fällen eine lokale Zirkulationsstörung angenommen hat. Der Unterschied beruht hauptsächlich in der Lokalisation. Ursprünglich lokalisierte Charcot*) die Entstehung der Lähmmung bei der ophthalmoplegischen Migräne in die basalen Oculomotoriuszentren, and zwar supponierte er hier denselben Prozess wie schon vorher bei der ophthalmischen Migräne ${ }^{24}$ ) in der Gehirnrinde: eine lokale transitorische Anämie infolge Gefässspasmus. Charcot hat diese ursprüngliche Erklärung später selbst wesentlich modifiziert, und wir sollten daher vielleicht gleich zu dieser zweiten Erklärung übergehen. Aber ich halte es doch für notwendig, auch auf die ursprüngliche Theorie Charcots näher einzugehen, weil in der letzten Zeit Seiffer ${ }^{6}$ ) und Leclézio'11) sich offen zu derselben bekennen. Auch Oppenheim ${ }^{4}$ ) und Edinger ${ }^{25}$ ) sprechen sich über die ophthalmoplegische Migräne im wesentlichen ganz analog aus wie die ursprüngliche Theorie Charcots.

Die Gründe, die gegen diese Theorie sprechen, sind doppelter Art: einerseits lässt sich die vasokonstriktorische Natur, andererseits die Lokalisation in die Basalzentren des Oculomotorius bestreiten.

Selbst wenn wir die vasomotorische Grundlage der Migräne überhaupt nicht in Abrede stellen wollen, ist es dennoch unwahrscheinlich, dass die Oculomotoriuslähmung in einer transitorischen Anämie eines bestimmten Gefässdistriktes im Zentralnervensystem beruhen sollte. Schon die vasokonstriktorische Erklärung der ophthalmischen Migräne steht auf schwachen Füssen. Gowers und Möbius haben durch zahlreiche überzeugende Gründe gezeigt, dass es nicht möglich sei, den ganzen Charakter und Verlauf des Migräneanfalls, speziell die verschiedenen Arten der Aura durch eine einfache Anämie der Gehirn-

*) Das Original dieser ursprünglichsten Erklärung konnte ich in den Schriften Charcots, insofern sie in dieser Abhandlung verzeichnet sind, nicht finden. Leclé zio ${ }^{11}$ ) aber zitiert sie in ausführlicher Weise ohne nähere Quellenangabe. 
rinde oder überhaupt des Zentralnervensystems zu erklären. Thomayer ${ }^{26}$ ) will zwar die Charcotsche Theorie der ophthalmischen Migräne nicht bestreiten, hat aber an derselben auszusetzen, dass bei anderen Körperteilen tüber pathologische, vom Arterienpasmus ausgehende Erscheinungen nur wenig bekannt sei.

Übrigens, selbst wenı die vasokonstriktorische Erklärung der ophthalmischen Migräne richtig wäre, liesse sich dieselbe nicht so ohne weiteres auf die ophthalmoplegische. Migräne übertragen. Mit Recht weisen Mingazzini ${ }^{9}$ ) und Ryba darauf hin, dass zwischen der ophthalmischen und ophthalmoplegischen Migräne der wichtige Unterschied bestehe, dass bei der ophthalmischen Form die Lähmungen vor den Kopfschmerzen auftreten, bei der ophthalmoplegischen Form dagegen nach den Kopfschmerzen. Heveroch ${ }^{10}$ ) meint, man dürfe die ophthalmische und ophthalmoplegische Migräne aus dem Grunde nicht neben einander stellen, weil sie anatomisch und klinisch einander fern stehen. Syllaba ${ }^{10}$ ) ist der Ansicht, die Charcotsche Theorie der ophthalmischen Migräne eigne sich aus dem Grunde nicht für die ophthalmoplegische Migräne, weil bei dieser die Lähmung unverhältnismässig länger dauere, ein Umstand, der einen Gefässspasmus ausschliesse.

Wenn wir uns die Oculomotoriuslähmung als Folge einer lokalen Anämie des basalen Zentrums vorstellen, dann entsteht die Frage, warum gerade dieses Zentrum von den übrigen allein und ausschliesslich in Mitleidenschaft gezogen sein soll, warum die Lähmung während des ganzen Lebens einseitig bleibt, obwohl es bekannt ist, dass die ophthalmische Migräne die Seite wechselt.

Bernheimer ${ }^{8}$ ), der sich viel mit der Histologie und Physiologie der Oculomotoriuszentren befasst hat, behauptet, dass das Zentrum für den Sphinkter iridis (kleinzelliger medialer Kern) aus einem ganz anderen Gefässbezirke (dem dorsolateralen Bezirk des Pedunculus) versorgt werde, während alle übrigen Muskeln, resp. Oculomotoriuskerne (inklusive die Akkomodation) ihre eigenen Äste direkt aus der Arteria cerebri posterior beziehen. Übrigens stimmen auch alle anderen Autoren ${ }^{8}$ ), welche den Blutkreislauf in den Oculomotoriuskernen histologisch untersucht haben (Heubner, Duret, d'Astros und Alzais, Schimamura, Marina, Oppenheim), wenn anch ihre Angaben in der näheren Beschreibung von einander abweichen, darin überein, dass der ganze Komplex der Oculomotoriuskerne nicht von einem einzigen Gefäss allein versorgt werde. Da aber bei der ophthalmoplegischen Migräne der ganze Oculomotorius, inklusive der Innenmuskulatur, in Mitleidenschaft gezogen ist, könnten wir uns den 
Gefässkrampf in einem derartigen vasomotorisch nicht einheitlichen Bezirk wohl schwerlich erklären.

Gegen die nukleäre Lokalisation der periodischen Oculomotoriuslähmung werden ausserdem noch folgende gewichtige Gründe angeführt:

Die echte periodische Oculomotoriuslähmung bleibt nur auf einen Oculomotorius beschränkt, während die trophischen Prozesse beiderseitig zu sein pflegen und die Kerne der Innenmuskulatur intakt lassen.

Bei den bis jetzt sezierten Fällen von periodischer Oculomotoriuslähmung (S. 206) waren die Oculomotoriuskerne entweder vollständig oder fast vollständig intakt, wenigstens was die Zellen anbelangt. Die relative Abnahme der Fasern im Falle von Karplus lässt sich durch Degeneration der betreffenden Nerven infolge einer groben periripherischen Störung und langjähriger Inaktivität exklären.

Schmidt-Rimpler weist darauf hin, dass bei der periodischen Oculomotoriuslähmung auch der M. obliquus inferior derselben. Seite gelähmt zu sein pflegt, obzwar nach unseren heutigen Kenntnissen dieser Muskel sein Zentrum auf der anderen Seite der Kerne besitzt.

Fragen wir uns nun, wohin wir ausserhalb der Kerne die Ursache der Oculomotoriuslähmnng lokalisieren sollen, so entfällt a priori die cortikale und cerebrale Lokalisation überhaupt wegen der Form der (nur auf einen Nerven beschränkten) Lähmung. Auch die Lokalisation in der nächsten Umgebung des Oculomotoriuskerns, d. i. im Pedunculus cerebri, ist schon von vornherein ausgeschlossen, weil hier so zahlreiche und wichtige Bahnen dicht neben einander verlaufen, dass ihr gleichzeitiges Ergriffensein früher oder später die periodische Oculomotoriuslähmung komplizieren müsste.

Also schon auf dem Wege der Exklusion sind wir gezwungen, die Ursache der periodischen Oculomotoriuslähmung irgendwo im Nerven selbst nach dessen Austritt aus dem Zentralnervensystem zu suchen.

Zu dieser Überzeugung gelangten mit der Zeit Möbius und Charcot, obzwar beide die kausale Störung der periodischen Lähmung ursprünglich in die nukleäre Gegend des Oculomotorius lokalisierten. Diese modifizierten Erklärungen der beiden Autoren stehen einander auch in den Details sehr nahe, denn so, wie später Möbius, nahm schon damals Charcot*) eine periodische lokale "Fluxion" im basalen Ver-

*) Charcot, Clinique des maladies du système nerveux (publ. par Guinon). Paris 1892. T. I. p. 87. 88; ... il s'agirait là (dans les cas de migraine 
lauf des Oeulomotorius an (die mit der Zeit in eine Entzündung übergehen soil und dadurch die Lähmung stabilisieren kann); ausserdem spricht er auch von einer Reizung der Trigeminusfasern und Übergang der Störung auf die Meningen.

Wir können aber gleich im vorhinein sagen, dass uns zur Lokalisation der Ursache der periodischen Oculomotoriuslähmung irgendwo an der Basis cranii nicht bloss der Weg der Exklusion führt, sondern auch gewisse objektive Symptome, die in typischen Fällen von ophthalmoplegischer Migräne beobachtet wurden.

Darkschewitsch ${ }^{9}$ ), Vissering, Kollarits ${ }^{38}$ ) und Karplus ${ }^{30}$ ) beobachteten auf der kranken Seite entweder eine Hypo- oder Hyperästhesie im Bereiche des 1. Trigeminusastes während des Anfalls. Im Fall Visserings, Rybas ${ }^{12}$ ) und in unserem eigenen Falle liess sich im Anfall Odem der Lider und mässiger Tränenfluss auf der gelähmten Seite konstatieren; in Rybas ${ }^{12}$ ), Russels ${ }^{11}$ ) und unserem Falle Injektion der Bindehaut des ergriffenen Auges; in unserem Falle sowie in den Fällen von Molon ${ }^{27}$ ), Lafon und Villauonte ${ }^{1 I}$ ) ein mässiger Exophthalmus des gelähmten Auges. Vom Herrn Dozenten Matys, der unseren Fall mehrmals im Anfall und ausserhalb desselben untersuchte, wurde im Anfall eine stärkere Füllung und Pulsation der Netzhautvenen des gelähmten Auges konstatiert. Auch $\mathrm{Manz}^{23}$ ) und Möbius ${ }^{1}$ ) erwähnen eine stärkere Schlängelung der Venen des ergriffenen Auges.

Der wichtigste Beweis für eine basale Oculomotoriusstörung ist aber der Sektionsbefund in zwei typischen Fällen von ophthalmoplegischer Migräne, welche nach vieljähriger Dauer dieser Migräne in höherem Alter zur Sektion kamen. Diese beiden Fälle sind fast in allen grösseren Abhandlungen über die ophthalmoplegische Migräne genau zitiert und daher dürfte es genügen, wenn wir hervorheben, dass - soweit die periodische Oculomotoriuslähmung in Betracht kommt - in beiden Fällen nichts anderes gefunden wurde als ein ganz kleiner benigner Tumor (Thomsen-Richter ${ }^{28}$ ): Fibrochondrom; Karplus ${ }^{29}$ ): Neurofibrom), der - was auffallend ist - in beiden

ophthalmoplégique) de simples "fluxions" avec excitations puis paralysie vasomotrice consécutive... Les douleurs de la periode hémicranique établissent suffisamment une participation de la cinquième paire ... Lingi c'est sur le trajet periphérique, basilaire, que porte la lésion tantôt transitoire, tantôt permanente du nerf oculomoteur et l'on conçoit, que dans ce dernier cas, elle puisse s'étendre aux parties avoisinantes de méninges.

*) Dasselbe wurde beobachtet in den Fällen von Fürst9), Mingazzini ${ }^{9}$ ), Cautalamessa ${ }^{9}$ ), Kljatschkin ${ }^{11}$ ), Giebler ${ }^{9}$ ), doch darf man auf diese nicht verweisen, weil sie nicht typisch sind. 
Fällen im Oculomotorius gerade dort lokalisiert war, wo dieser Nerv in die Dura mater eintritt.

Anfangs hielten Charcot und Möbius den Befund Richters für einen zufälligen und vermuteten, der Tumor sei in dem bereits aus einer anderen Ursache lädierten Nerven entstanden. Später, als Charcot die periodische Oculomotoriuslähmung als Folge einer lokalen "Fluxion" im basalen Verlauf des N. oculomotorius erklärte, nahm er an, dass die sich öfters wiederholende Zirkulationsstörung die Entstehung sekundärer organischer Veränderungen in dieser Gegend, resp. im N. oculomotorius selbst unterstütze. Als aber Karplus einen ähnlichen Befund wie Richter beschrieb, da änderte Möbius seine Ansicht total und schreibt nun (S. 200) diesen benignen Geschwülstchen des Oeulomotorius eine aktive, primäre Aufgabe bei dem Anfall zu. Trotzdem erklären neuerdings Seiffer und de Lapersonne die beiden erwähnten Sektionsbefunde wiederum für Zufälle, was aber an und für sich nur wenig wahrscheinlich ist, weil es doch ein ganz besonderer Zufall wäre, wenn in den einzigen zwei sezierten Fällen von ophthalmoplegischer Migräne der Befund ganz genau derselbe wäre.

Aus den obigen Ausführungen ist ersichtlich, dass die Ansichten der Autoren über die Bedeutung der gefundenen benignen Geschwülstchen des Oculomotorius wesentlich von einander abweichen, und deshalb erlaube ich mir, im Folgenden etwas ausführlicher auf meine eigene Erklärung der Entstehung dieser Geschwülstchen bei der ophthalmoplegischen Migräne einzugehen.

Das Ödem der Lider, die Injektion der Bindehaut, die Füllung der Venen, der Exophthalmus (S. 206) deuten auf eine Störung des venösen Kreislaufs irgendwo in der Tiefe hinter dem Bulbus, und zwar am wahrscheinlichsten im Sinus cavernosus; damit würde auch die Läsion des 1. Trigeminusastes übereinstimmen. Nun lagen aber die gefundenen Geschwülstchen des Oculomotorius intradural, d. i. an der Eintrittsstelle dieses Nerven in die Dura, direkt über dem Sinus cavernosus; scheinbar würde also dieser Befund mit einer Läsion des Sinus cavernosus, der extradural liegt, nicht zusammenhängen.

Lassen wir diesen scheinbaren Widerspruch vorerst beiseite und fragen wir uns, welche Veränderungen sich im Sinus cavernosus bei einem Anfall dox ophthalmoplegischen Migräne abspielen dürften. Sicherlich dürfte es sich um einen von der nächsten Nachbarschaft ausgehenden Druck handeln, denn ein Hindernis im Sinus selbst (Thrombose) könnte nicht in kurzer Zeit beseitigt werden. Keinesfalls äussert sich dieser Duck nur innerhalb des Sinus selbst (als Blutstauung), sondern überträgt sich auch auf die Umgebung. Diese ist 
aber auf allen Seiten von festem unnachgiebigem Knochen umgeben und nur oben breitet sich die Dura mater aus, die sich hier zu beiden Seiten der Sella turcica über der S-förmig gekrümmten Carotis, dem Sinus cavernosus und dem sich verästelnden N. trigeminus als eine derbe, gespannte Membran wölbt. Fast in der Mitte dieser Wölbung tritt der N. oculomotorius durch die Dura mater. Da dieser Nerv mit der beschriebenen Wölbung der Dura - vor und nach seinem Durchtritt - fast parallel verlänft, kann ex dieselbe nicht in Form eines einfachen Loches durchbohren, das in dieser Wölbung einen von oben nach unten geöiffneten runden Defekt bilden würde, sondern in Form einer Öffnung, die sich von hinten nach vorn öffnet, was in der in derselben Richtung sich ausbreitenden Membran in der Weise geschieht, dass sich die Dura im Bereiche dieser Öffnung in zwei Blätter spaltet. Auf diese Weise entsteht in der Dura mater eine Spalte mit einer oberen und unteren Membran und diese Spalte benützt der N. oculomotorius zu seinem Durchtritt. Die harte Hirnhaut verwächst nicht gleich am inneren Rand der Spalte mit dem Nerv, sondern etwas weiter davon, so dass diese Öffnung eigentlich eine trichterförmige, nach rückwärts gegen das Gehirn geöffnete Form besitzt. Das untere Blatt endet also nicht dort, wo das obere Blatt beginnt, sondern erstreckt sich zum Teil unter dieses und wird allmählich dünner. Dagegen endet das obere Blatt über der Öffnung mit einem freien, derben Rand, der sich über den Oculomotorius von einer Seite der Öffnung zur anderen bogenförmig spannt*).

Wenn nun unterhalb dèr Wölbung der Dura ein Druck entsteht, so kann derselbe infolge der knöchernen Umgebung nur die Dura allein empordrängen, d. h. spannen. Dadurch muss notwendigerweise der N. oculomotorius leiden, da teils infolge Spannung der Dura die beiden Blätter der für den N. oculomotorius bestimmten Öffnung sich aneinanderlegen werden, teils infolge des Drucks von unten das untere schwächere Blatt nachgeben und der N. oculomotorius gegen den derben Rand des oberen Blattes gedrückt werden wird.

Diese Spannung des Duragewölbes kann zwar nicht eine solche Kraft haben, dass etwa der $\mathrm{N}$. oculomotorius vollkommen gequetscht würde; dennoch aber können bei längerer Dauer eines solchen, wenn auch mässigen Drucks im Nerven gewisse Veränderungen entstehen, so dass die Leitungsfähigkeit, wenn nicht ganz und gar unterdrückt,

*) Von diesen anatomischen Verhältnissen habe ich mich gelegentlich klinischer Sektionen mehrmals selbst überzeugt. Übrigens geben Wilbrand und Saenger (l. c. S. 316) dieselbe Beschreibung des Durchtrittes des N.oculomotorius durch die Dura mater. 
so doch wenigstens vermindert sein kann. Übrigens lehrt auch der klinische Verlauf der ophthalmoplegischen Migräne, dass die Läsion, an der der N. oculomotorius bei der ophthalmoplegischen Migräne leidet, eine sehr labile und leichte ist. Der Schmerz muss mindestens 1-2 Tage dauern, damit überhaupt bestimmte Symptome der Lähmung eintreten. Nach leichten Anfällen bessert sich die Lähmung gleich nach dem Aufhören der Schmerzen. Die Anfälle wiederholen sich manchmal 20-30 Jahre, bevor eine vollständige Lähmung des Oculomotorius eintritt.

Aus dem Angeführten geht mit grosser Wahrscheinlichkeit hervor, dass der N. oculomotorius bei jedem Anfall von ophthalmoplegischer Migräne an seiner Durchtrittsstelle durch die Dura komprimiert, resp. mechanisch gereizt wird. Und wenn wir nun mit dieser Erklärung den Befund benigner Geschwülstchen (von der Grösse einer halben Erbse), die in den beiden sezierten Fällen von ophthalmoplegischer Migräne am N. oculomtorius gefunden wurden, vergleichen, dann werden wir es begreifen, warum die Geschwülstehen in beiden Fällen unmittelbar an der Dura mater lagen, d. i. an jener Stelle, wo der Nerv in seine Öffnung in der harten Hirnhaut eintritt und wo sich daher jene zarte Quetschung des Nerven fortwährend wiederholt.

Unter diesen Umständen, wo sich die Quetschung, sei es auch in zarter Weise, öfters wiederholt resp. längere Zeit dauert, reagieren alle unsere Organe (namentlich die Haut) und daher vielleicht auch die Nerven in der Weise, dass an einer derartigen Stelle eine Schwiele entsteht, d. i. jenes benigne Geschwülstchen, das in dem einen Falle als Fibrochondrom, in dem anderen Falle als Neurofibrom bezeichnet wurde. Offenbar konnte sich diese "Schwiele" nur in der zentripetalen Partie des N. oculomotorius entwickeln, da es sich um einen motorischen Nerv handelt, von dem bekannt ist, dass sein zweiter (rom Zentrum abgetrennter) Teil mehr oder weniger einer vollständigen Degeneration unterliegt; andererseits hat vielleicht auch der Druck, der gerade unter der Dura am meisten gewirkt hat, zugleich eine übermässige Reaktion (Wucherung des Bindegewebes) unterdrückt.

Auf diese Weise erklärt es sich zugleich, warum gerade der N. oculomotorius relativ so häufig von benignen Geschwülstchen, die Möbius für angeboren hält, heimgesucht wird. Die Erklärung liegt darin, dass die Geschwülstchen nicht echt, d. i. angeboren, sondern einfache, erworbene Schwielen und daher immer so klein sind. In den übrigen Gehirnnerven können sie nicht vorkommen, wenigstens nicht so häufig, weil der Durchtritt der übrigen Gehirnnerven durch die Dura anders geformt ist als beim N. oculomotorius.

Wie aus dem dritten, weniger typischen Fall von sezierter ophthal- 
moplegischer Migräne hervorzuheben scheint, reagiert der N. oculomotorius nicht immer auf die beschriebene periodische Iäsion durch eine geschwulstartige Verdickung, wiewohl hierdurch der Verlauf dieser Krankheit in keiner Weise geändert wird. Weiss ${ }^{30}$ ) beschrieb nämlich einen Fall von periodischer Oculomotoriuslähmung, die oft wiederkehrte und laut Anamnese seit der Kindheit datierte. Dieser Fall hat aber den Fehler, dass sich in der Krankheitsgeschichte keine Erwähnung darüber findet, ob die Lähmungsanfälle von Kopfschmerzen begleitet waren. Da die Beschreibung dieses Falles überhaupt nur kurz und oberflächlich ist, lässt sich die Möglichkeit nicht ausschliessen, dass es sich dennoch um eine echte ophthalmoplegische Migräne gehandelt hat, und da müssen wir auch mit dem Sektionsbefund am N. oculomotorius rechnen, der erhoben wurde, nachdem die betreffende Person infolge vorgeschrittener Tuberkulose gestorben war. Weiss fand den N. oculomotorius abgeflacht, grau verfärbt, die von ihm versorgte Muskulatur degeneriert. An der Austrittsstelle des N. oculomotorius aus dem Gehirn fanden sich zahlreiche tuberkulöse Granulationen von Mohnkorngrösse. Weiss erblickt in diesen Granulationen die Ursache der Lähmung; aber es ist klar, dass diese Granulationen kaum schon von Jugend auf bestanden und dass sich auch die drei rein transitorische Anfälle von totaler Oculomotoriuslähmung, die der Autor selbst im Verlauf von etwa 3 Monaten beobachtet hatte, schwerlich durch dieselben erklären lassen. Eher ist es wahrscheinlich, dass diese Grranulationen in loco minoris resistentiae, i. e. in dem bereits lädierten Nerven entstanden sind. Auf eine periodische Lähmung weist eher jene Abflachung des Oculomotorius hin, die wir durch den oben angegebenen Mechanismus bei der ophthalmoplegischen Migräne bequem erklären können, obwohl es diesmal nicht zur Bildung eines Geschwülstchens gekommen ist.

Die benachbarten Gehirnnerven (IV., V., VI.), die in der nächsten Nachbarschaft des N. oculomotorius ebenfalls die Dura durchbohren, unterliegen der ophthalmoplegischen Migräne gewöhnlich nicht, da ihr Durchtritt an der Peripherie jener Wölbung erfolgt, die sich über dem Sinus cavernosus ausbreitet und zwar entweder dort, wo die Dura wiederum am Knochen inseriert (V. und VI.) oder dort, wo sie in das Tentorium cerebelli übergeht (IV.). Bei der Schwellang des Sinus cavernosus oder seiner nächsten Umgebung und beim Empordrängen der Durawölbung müssen sich ihre Durchtrittsöffnungen eher erweitern.

Die peripheren Nerven können in eine ähnliche Situation nie gelangen und daher wird eine periodische Lähmung bei ihnen nie 
beobachtet, obzwar sie dieselbe Eignung für angeborene Geschwülste besitzen.

Im Falle Charcots war zwar ausser dem N. oculomotorius auch der N. abducens ergriffen, ja in den Fällen Brissauds, Sciamanas and Chabberts betraf die rezidivierende Lähmung alle drei motorische Augennerven (N. oculomotorius, abducens, trochlearis), es ist aber fraglich, ob es sich in diesen Fällen um eine typische ophthalmoplegische Migräne gehandelt hat, denn andere Autoren rechnen diese Fälle entweder nicht hierher oder lehnen sie überhaupt ab (S. 187). Nur der erste Ast des Trigeminus bot, wie ich bereits erwähnt habe, in einigen sonst verbürgten Fällen von ophthalmoplegischer Migräne gewisse Störungen der Sensibilität.

Ähnlich verhält sich die Sache mit jenen Fällen, welche als isolierte periodische Lähmung des $\mathrm{N}$. abducens oder trochlearis geführt werden. Bernheimer ${ }^{8}$ ) z. B. giebt an, dass er, Ormerod and Marina solche Fälle, die nur den N. abducens betrafen, beobachtet haben. Doch muss hinzugefügt werden, dass Bernheimer die periodische Lähmung mit der (einige Male) rezidivierenden Lähmung überhaupt verwechselt.

Nur der Fall Luzenbergers ${ }^{31}$ ), auf welchen auch Möbius hinweist, hat in der Tat eine fast auffallende Ähnlichkeit mit der ophthalmoplegischen Migräne, obwohl nicht der N. oculomotorius, sondern ausschliesslich nur der N. trochlearis ergriffen war. Die Mutter litt an Migräne. Der erste Anfall stellte sich im 12. Lebensjahre ein und damals dauerten die Migräne und die Diplopie nur einen Tag; die späteren Anfälle waren zwar weniger häufig, aber von längerer Dauer, sowohl was die Schmerzen als auch was die Lähmung anbelangt. Einen der grössten Anfälle beobachtete der Autor selbst, als der Patient bereits 29 Jahre alt war. Der Kopfschmerz dauerte 5 Tage, die Lähmung noch weitere 15 Tage. Die genaue Untersuchung der Diplopie ergab, dass es sich um eine Trochlearislähmung handelte. Da die rechte $V$. temporalis abnorm gefüllt war, so nahm der Autor an, dass die Ursache der Anfälle in einer periodisch wiederkehrenden Schwellung des Sinus cavernosus lag.

Im Prinzip kann man also nicht in Abrede stellen, dass ausnahmsweise auch bei der reinen ophthalmoplegischen Migräne ausser dem $\mathrm{N}$. oculomotorius auch der $\mathrm{N}$. abducens oder trochlearis gleichzeitig oder selbständig beteiligt sein kann. Selbst der Oculomotorius kann, wie ich bereits erwähnt habe, die Migräne nur auf Grund einer besonderen lokalen anatomischen Disposition schädigen, und man kann daher mit demselben Rechte eine ähnliche Disposition auch für denN. abducens oder trochlearis annehmen. Ich glaube nicht, dass die 
von mir weiter oben gegebene Erklärung der Oculomotoriuslähmung bei der ophthalmoplegischen Migräne die einzig mögliche sei, sondern bin der Ansicht, dass sie die gewöhnlichste von allen sei, die vorkommen können. Daneben ist es aber immer noch möglich, dass nach einer lokalen Meningitis, Periostitis, oder bei einem Aneurysma, Tumor u. drgl., eventuell bei einem abnormen Verlauf der betreffenden Nerven in der Gegend des Sinus cavernosus derartige anatomische Veränderungen entstehen können, dass bei einem symptomatischen oder idiopathischen Migräneanfall gleichzeitig mit dem N. oculomotorius oder unabhängig von diesem der N. abducens resp. trochlearis leidet.

Von mehreren Seiten (Bernheimer, Weigner ${ }^{10}$ ) u. a.) wixd beispielsweise angegeben, dass alle 3 motorischen Augennerven so eingeengt zwischen Carotis und Dura mater verlaufen, dass eine geringe Erweiterung dieser Arterie oder eine Lageveränderung derselbęn die genannten Nerven, speziell den N. oculomotorius gegen die harte, gespannte Dura andrücken muss. Auch dort, wo diese Nerven die derbe, feste, die Fissura orbitalis superior verschliessende Membran durchsetzen, können dieselben in ihren Kanälchen, sei es nun durch Ödem, Hyperämie oder durch eine ektatische Vene, eingeklemmt werden*).

Auch die Läsion des 1. Trigeminusastes, die in mehreren Fällen von ophthalmoplegischer Migräne beobachtet wurde, weist darauf hin, dass eine lokale Störung der Augennerven bei dieser Krankheit auch subdural, d. i. bereits nach dem Durchtritt durch die Dura entstehen kann. Hierbei dürfen wir allerdings nicht vergessen, dass eine subdurale Läsion des N. trigeminus keineswegs eine gleichzeitige Einklemmung des $\mathrm{N}$. oculomotorius in seiner Duralöffnung ausschliesst.

Infolge des so sehr komplizierten Verlaufes der Augennerven an der Basis cranii lassen sich nicht alle möglichen Kombinationen aufzählen, die unter dem Bilde der ophthalmoplegischen Migräne verlaufen können, aber die beiden sezierten Fälle beweisen, dass der Durchtritt des $\mathrm{N}$. oculomotorius durch die Dura mater jene Stelle sein dürfte, wo in den typischen, vielleicht könnte man sagen idiopathischen Fällen dex ophthalmoplegischen Migräne der Nerv lädiert ist.

Nachdem wir die Stelle bestimmt haben, wo die ophthalmoplegische Migräne entsteht, würde uns noch erübrigen zu entscheiden, worin die supponierte Drucksteigerung in der Gegend des

*) Nach Bernheimer haben der N. trochlearis und der 1. Ast des Trigeminus je einen eigenen Kanal, währead der N. abducens, oculomotorius und die $V$. ophthalmica einen gemeinsamen Kanal haben. 
Sinus cavernosus besteht und wie sie zustande kommt. Diesbezüglich müssen wir uns vorläufig mit mehr oder weniger wahrscheinlichen Vermutungen begnügen, da eine sichere Begründung mangels sezierter Fälle vorläufig unmöglich ist.

Aus dem ganzen Verlauf der ophthalmoplegischen Migräne und gewöhnlichen Migräne, namentlich aber aus dem vollständig negativen anatomischen Befund bei der letzteren müssen wir schliessen, dass das Wesen der genannten Drucksteigerung in einer Funktionsstörung beruhe. Wahrscheinlich schwillt ein Organ in der Nachbarschaft des Sinus cavernosus oder vielleicht nur eine Öffnung, eventuell nur überhaupt eine bestimmte Stelle an, wodurch ein gewisser Druck entsteht, der durch Störung des venösen Kreislaufs im Sinus cavernosus noch steigen muss. Diese Schwellung kann ihre Ursache in einer inneren Sekretion haben oder in einer ödematösen Durchtränkung, oder sie ist die Folge einer lokalen Hyperämie und eventuell handelt es sich um eine Kombination aller dieser Prozesse.

Von diesen Möglichkeiten ist am meisten berechtigt die Vermutung einer lokalen Hyperämie, sei es nun infolge einer lokalen oder zentralen Reizung der Vasomotoren. Zu dieser Überzeugung gelangte nicht nur Charcot, sonderm auch Möbius und taberhaupt die Mehrzahl der Autoren, die sich mit der ophthalmoplegischen Migräne beschäftigt haben. Nur durch eine lokale Hyperämie können wir uns die Labilität der Migräneanfälle und ihre Reaktion auf Antipyretica erklären; bekanntlich wirken diese Präparate stark auf die Vasomotoren.

Hiermit kehren wir zu der alten Ansicht der Autoren zurück, man könne die Migräne durch eine vasomotorische Reaktion erklären, einer Ansicht, zu der sich bis jetzt eine grosse Anzahl der Neurologen bekennt, mögen auch ihre Meinungen in den Details teilweise von einander abweichen (Oppenheim, Edinger, Schmidt-Rimpler u. a.). Übrigens bestreitet weder Gowers noch Möbius ganz und gar die Mitbeteiligung der Vasomotoren beim Migräneanfall. Ihre Migränetheorie vermag nicht das Hauptsymptom der Migräne, d. i. den Kopfschmerz zu erklären und sie nehmen in diesem Punkte ihre Zuflucht wiederum zu vasomotorischen Störungen, die sie in die Hirnhäute einer Gehirnhälfte lokalisieren. Auch bei der ophthalmoplegischen Migräne gibt Möbius ausdrücklich eine lokale Hyperämie als Ursache der Schmerzen an.

Forschen wir nun in der Nachbarschaft des Sinus cavernosus nach einem Organ oder einer Prädilektionsstelle, die vasomotorischen Veränderungen ausgesetzt sein könnte, dann muss in erster Reihe die Hypophysis cerebri unsere Aufmerksamkeit auf sich lenken. Dieses 
Organ besteht bekanntlich aus zwei Teilen, von denen der eine, grössere, sicher einen Drüsencharakter besitzt und reich an Gefässen ist. Schon aus diesem Umstande können wir schliessen, dass diese "Drüse" entsprechend den Zirkulationsänderungen ihren Umfang bedeutend verändern kann.

Die Idee, dass eine temporäre Schwellung der Hypophysis den Migräneanfall bedingen könnte, hat schon vor mehreren Jahren De yl ${ }^{32}$ ) ausgesprochen. Durch die anatomische Untersuchung der lokalen Verhältnisse dieser Drüse hat er sich überzeugt, dass bei den meisten Leichen zwischen Arteria carotis und Hypophysis ein freier Raum vorhanden ist. Bei mehreren Fällen aber, die bei Lebzeiten an Migräne gelitten hatten, war diese Drüse vergrössert und berührte die Arteria carotis, ja füllte manchmal sogar den Bogen, den dieselbe an dieser Stelle bildet, aus. Einen analogen Befund erhob er oft auch bei Leichen aus der Irrenanstalt, woraus man schliessen darf, dass die Vergrösserung der Zirbeldrüse, analog der Migräne selbst, mit einer neuropathischen Belastung eng zusammenhängt.

Die Migräneanfälle entstehen nach Deyls Ansicht dadurch, dass die an und für sich schon vergrösserte Hypophysis periodisch anschwillt und die Carotis drückt, wodurch zwischen Carotis und Dura der 1. Ast des Trigeminus komprimiert wird. Dieser Effekt, resp. die dadurch entstehenden Schmerzen steigern sich nach Deyls Erklärung noch durch die Venostase, die infolge der partiellen Kompression des Sinus cavernosus in den zuführenden Bahnen entsteht. Auf diese Weise setzt er auch eine Störung im Sympathicus voraus, leitet aber davon nur die vasomotorischen Störungen ab, die den Migäneanfall oft begleiten. Die Besserung der Migräne im Alter erklärt er durch Verkleinerung und kolloide Degeneration der Hypophysis im Alter.

Von grosser Bedeutung für die Erklärung der ophthalmoplegischen. Migräne als Folge einer Hypophysisschwellung sind die Fälle von Tumoren der Hypophysis oder Tumoren der Sella turcica oder deren nächsten Umgebung überhanpt. Bernheimer sagt von den Geschwülsten dex Hypophysis, dass sie unter den Augennerven am häufigsten den $\mathrm{N}$. oculomotorius schädigen und zwar entweder direkt. durch den Druck oder manchmal nur durch Fernwirkung ohne direkten mechanischen Zusammenhang. Sternberg ${ }^{33}$ ) führt an, dass eine Lähmung des N. abducens oder trochlearis bis jetzt bei der Akromegalie nicht beobachtet wurde, die Oculomotoriuslähmung aber ein häufiges Symptom derselben sei. Diese Lähmung muss nicht alle vom N. oculomotorius versorgte Muskeln betreffen (Sternberg, Bruns u. a.) und kann wieder zurückgehen (Mosler ${ }^{33}$ ), Schlesinger ${ }^{33}$ ), Wilbrand und Saenger). Ein regelmässiges Symptom bei der 
Akromegalie (resp. den Tumoren der Hypophysis) sind Kopfschmerzen*), die gewöhnlich in Anfällen auftreten und manchmal mit Erbrechen einhergehen, so dass sie vollständig den Charakter der echten Migräne an sich tragen (Sternberg, Deyl u. a.). Sternberg macht darauf aufmerksam, dass nach einem solchen Anfall oft eine Abnahme der Sehschärfe, Exophthalmus oder eine Oculomotoriuslähmung zurückbleibt.

Wie sehr Hypophysistumoren eine ophthalmoplegische Migräne vortäuschen können, erhellt auch aus einem eigenen Fall, den wir heuer in der Klinik des Herrn Prof. Maixner beobachtet haben. Es handelte sich um ein Sarkom, das, wie die Sektion zeigte, gerade aus dem Zentrum des Keilbeins hervorwuchs und seine Umgebung ebenso. wie ein Tumor der Hypophysis beeinflusste. Die ganze Krankheit dauerte seit ihrem Beginn etwa ein halbes Jahr. Während der ersten 6 Wochen empfand der Kranke nur fortwährende, zeitweise exazerbierende Kopfschmerzen, u. zw. nur in der linken Kopfhälfte; ihr Maximum war in der Tiefe der Orbita. Dann erst zeigte sich eine Parese des N. abducens und oculomotorius derselben Seite, die nach fünftägiger Dauer für einige Tage verschwand. Als sie aber wiederkehrte, ging sie rasch in eine dauernde Lähmung über. Da erst gingen die Schmerzen auch auf die andere Kopfseite über und auch hier entwickelte sich (binnen 4 Wochen) eine Paralyse derselben Nerven wie auf der linken Seite. Erst im weiteren Verlauf stellte sich eine Einschränkung des Gesichtsfeldes und eine totale beiderseitige Ophthalmoplegie mit starkem Ödem der Lider und der Bindehänte ein.

Wie man sieht, kommen alle Symptome der ophthalmoplegischen Migräne auch bei den Tumoren der Hypophysis, resp. bei der Akromegalie vor. Ja sogar die Periodizität der Schmerzen und der Lähmung pflegt manchmal teilweise angedeutet zu sein.

In wie weit die vergrösserte Schilddrüse in unserem (eingangs beschriebenen) Falle auf eine gleichzeitige Anomalie der Hypophysis hindeutet, will ich nicht entscheiden; aber so viel ist sicher, dass bei Akromegalie regelmässig gleichzeitige Veränderungen in beiden Drủsen gefunden wurden.

Ich bin mir dessen wohl bewusst, dass das Verhältnis zwischen Hypophysisschwellung und Migräne überhaupt, der ophthalmoplegischen

*) Von einer Läsion des Trigeminus können diese Schmerzen nicht abgeleitet werden. Der Trigeminus ist unter den Augennerven von der Hypophysis am weitesten entfernt und deshalb sind die Sensibilitätsstörungen im Bereiche des Trigeminus bei reinen Hypophysistumoren selten (Brans, Eulenburgs Enzyklop. 1895. Bd. 8. S. 691). 
insbesondere nicht so einfach ist und dass sich manche Einwendungen erheben lassen.

Zunächst ist die Hypophysis ein zentral gelagertes Organ, so dass man Symptome von beiden Seiten der Sella turcica erwarten sollte, während doch die ophthalmoplegische Migräne während des ganzen Lebens stets einseitig bleibt; die gewöhnliche Migräne ist in der Mehrzahl der Fälle ebenfalls einseitig und kann überdies die Seite wechseln.

Ferner lehrt die klinische Erfahrung, das die Hypophysistumoren noch früher und häufiger Sehstörungen (Hemianopsie u. a.) verursachen als eine Oculomotoriuslähmung und zwar durch direkten Druck auf das Chiasma, das dicht über der Hypophysis liegt, so dass es von derselben nur durch die Dura mater getrennt ist. Bei der ophthalmoplegischen Migräne kommen aber Sehstörungen nur selten vor.

Doch sind diese Einwendungen nicht so entscheidend, dass wir die Erklärung der Migräne durch Hypophysisschwellung fallen lassen müssten, und lassen sich auf eine einfache und wahrscheinliche Weise umgehen.

Bekanntlich erhält die Hypophysis ihre ernäbrenden Gefässe aus beiden Carotiden; ihr Gefässsystem stammt also von zwei verschiedenen Seiten. Dass die vasomotorische Reaktion auch nur einseitig sein kann, ist eine bekannte Erfahrung und es kann daher auch nur die eine Hälfte der Hypophysis durch aktive Hyperämie anschwellen und eine einseitige Migräne hervorrufen. Zugleich könnte man es sich leicht erklären, warum die Migäne bei ein- und demselben Individuum die Kopfseite wechseln kann und warum sie oft beiderseitig ist.

Bei der gewöhnlichen Migräne. fehlen optische Störungen nicht ganz; sie werden als Aura beschrieben, die stets nur eine kleine Weile vor dem Migräneanfall andauert; doch hat man in seltenen Fällen auch beobachtet, dass sie während des ganzen Migräneanfalls anhielt ${ }^{34}$ ). Hutchinson ${ }^{35}$ ) und Rampoldi ${ }^{35}$ ) beschrieben Fälle, wo nach dem Anfall eine totale Erblindung des einen Auges vorhanden war.

Deyl ${ }^{32}$ ) erklärt das szintillierende Skotom bei der Migräne durch Druck der schwellenden Hypophysis auf die ernährenden Arterien des Chiasma, eventuell die Anfangsteile der Nervi optici; durch den Druck entsteht Anämie, durch diese eine Ernährungsstörung resp. das szintillierende Skotom, das nach Deyl so lange dauert, bis durch Regelung des Kollateralkreislaufs auch die Ernährung dieser Stellen der optischen Bahnen geregelt ist. Je nach dem, ob der Druck mehr auf den Anfangsteil eines Nervus opticus oder auf das Chiasma selbst einwirkt, entsteht entweder ein monokuläres oder ein binokuläres Skotom. 
Dass das szintillierende Skotom seine Ursache in den peripheren Sehnervenbahnen, ja sogar auch in der Netzhaut selbst haben kann, ist eine schon längst von zahlreichen Autoren vertretene Ansicht. Zuletzt weist wiederum $J_{0} l l y^{36}$ ) nach, dass die zentralen oder beide Gesichtsfelạhälften betreffenden szintillierenden Skotome ihren Ursprung nur im Chiasma haben können, obwohl er im übrigen diese Skotome für identisch hält mit der ophthalmischen Migräne. Dass die szintillierenden Skotome, die sich nur in einem Auge abspielen, einen peripherischen, im Nervus opticus oder in der Retina beruhenden Ursprung haben, wird wohl allgemein anerkannt.

Wir finden also auch bei der gewöhnlichen Migräne Sehstörungen und zwar ebenso wie bei den Tumoren der Hypophysis entweder beiderseitig (was häufiger vorkommt) oder einseitig. Es ist klar, dass es bei der Migräne niemals zu einer dauernden Sehstörung kommen muss, weil der Druck bei derselben nur ein vorübergehender ist und gewiss nur auf eine kleinere Stelle (im Sehnerven) einwirkt.

Etwas komplizierter scheint das Verhältnis zwischen Hypophysisschwellung und ophthalmoplegischer Migräne zu sein. Diese ist in allen Fällen stets nur einseitig und optische Störungen werden bei derselben nur selten beobachtet, obzwar die Oculomotoriuslähmung und die Kompression des Sinus cavernosus dafür sprechen würden, dass die Hypophysisschwellung hier eine noch grössere sein müsse als bei der gewöhnlichen Migräne. Also reicht eine einseitige Hyperämie der Hypophysis zur Erklärung der ophthalmoplegischen Migräne nicht aus. Analog der gewöhnlichen Migräne müsste man öfters auf einen Fall von ophthalmoplegischer Migräne stossen, wo wenigstens der Schmerz, wenn nicht auch die Lähmung, auch auf der anderen Seite vorhanden wäre, und ein solcher, sonst typischer Fall von ophthalmoplegischer Migräne ist bis jetzt noch nicht beschrieben worden. Im Gegenteil, man hat regelmässig beobachtet, dass auch der Schmerz, wenn er den mit Lähmung kombinierten Anfällen nur einige Jahre voranging, in jedem Anfalle ausnahmslos einseitig war.

Ich habe schon früher erwähnt, dass diese Einseitigkeit der ophthalmoplegischen Migräne sowie der fast regelmässige Mangel der Erblichkeit und vielfach auch der neuropathischen Belastung überhaupt uns zu der Supposition gewisser lokaler anatomischer, sei es nun angeborener oder erworbener Verhältnisse zwingt, welche die. Entstehung der ophthalmoplegischen Migräne unterstützen. Gerade in diesen anatomischen Veränderungen müssen wir wohl die Ursache dafür suchen, warum die Hypophysis, obzwar sie de norma zentral gelagert ist, doch stets nur eine einseitige Affektion hervorzurufen vermag.

Allerdings kann man mir einwenden, dass die Migräne nicht eine 
lokale anatomische Erkrankung sei, sondern dass ihr Wesen tiefer im Organismus begründet sei (Intoxikation, neuropathische Veranlagung), und dass daher eine lokale anatomische Disposition noch nicht genügt, um eine Migräne hervorzurufen. Demgegenüber erlaube ich mir aber zu bemerken, dass wir die Funktion der Hypophysis noch nicht kennen.

Schon aus den verschiedenen klinischen und anatomischen Erfahrungen lässt sich schliessen, dass diese Drüse nicht einmal für den gesunden Körper ohne Bedeutung, ohne Funktion sei. So wie die Migräneanfälle ungleich stark sind, kann auch die Schwellung der Drüse verschieden gross sein, oder mit anderen Worten, es ist sehr wahrscheinlich, dass auch bei ganz gesunden Menschen diese Drüse zeitweise hyperämisch wird und ihre Funktion sich einstellt oder wenigstens zunimmt. So wie manche Frauen infolge besonderer lokaler Verbältnisse eine schmerzhafte Menstruation haben, obzwar diese Verhältnisse nicht die Ursache der Menstruation sind, ebenso ist es ganz gut möglich, dass eine normale, zeitweilige Schwellung der Hypophysis, auf dem Wege analoger Umstände, das Bild der ophthalmoplegischen Migräne herbeiführen kann. Zum Unterschied von dieser Migräne, wo die sonst normale, zeitweilige Schwellung der Hypophysis sich unter dem Einfluss lokaler anatomischer Veränderungen passiv steigern würde, würde es sich bei der gewöhnlichen Migräne um eine rein aktiv gesteigerte Schwellung der Hypophysis (infolge einer reuropathischen Veranlagung) handeln.

Dass die ophthalmoplegische Migräne die gleiche Periodizität einbält und die gleichen Eigenschaften besitzt wie die gewöhnliche Migräne (Reaktion auf Antipyretica, auf die Gravidität usw.), obwohl die lokale Disposition bei derselben die Hauptrolle spielt, kann uns nicht überraschen, wenn wir annehmen, dass bei beiden Migräneformen die schon de norma periodische oder wenigstens periodisch sich steigernde Funktion (resp. Schwellung) der Hypophysis die Grundlage der Anfälle bildet.

Die Erklärung der ophthalmoplegischen Migräne durch eine anatomische Disposition in der Gegend des Sinus cavernosus ist kein leeres Theoretisieren. Wenn wir die einzelnen publizierten Fälle dieser Krankheit durchgehen, so finden wir in den betreffenden Krankengeschichten im objektiven Befund verschiedene Bemerkungen, die dafür sprechen, dass diese Erklärung eine reelle Basis besitzt. Omerod und Spicer ${ }^{11}$ ) und Russel ${ }^{11}$ ) geben an, dass der N. opticus auf der kranken Seite etwas "atrophisch" war. Saundby ${ }^{9}$ ) und Seiamanna ${ }^{9}$ ) fanden die Umgebung der Papille verwischt; im letzteren Falle bestand ausserdem eine Chorioiditis in der Gegend der Fovea. Schilling ${ }^{37}$ ) 
sah im erkrankten Auge Bündel von Myelinfasern. In den Fällen Thomsen ${ }^{9}$ ), Hinde ${ }^{9}$ ) und Kollarits ${ }^{38}$ ) und in unserem Falle wurde eine beträchtliche Amblyopie des gelähmten Auges*) ohne ophthalmoplegischen Befund beobachtet. Thomsen fand überdies eine hochgradige konzentrische Gesichtsfeldeinengung; wir wiederum einen beträchtlichen Astigmatismus, ebenfalls nur auf der kranken Seite.

Aus diesen wenigen Fällen ist es sicherlich klar, dass bei der ophthalmoplegischen Migräne verhältnismässig oft gewisse angeborene oder auch erworbene Anomalien beobachtet werden, die stationär und nicht wie die Lähmung eine Folge des Anfalls sind. Dort, wo sich auffallend häufig sichtbare Anomalien einstellen, können wir gewiss mit vollem Recht auch innere Anomalien (der Hypophysis, des Sinus cavernosus u. dgl.) annehmen, zumal wenn es sich um eine benachbarte Stelle auf derselben Kopfhälffe handelt.

Bis jetzt hat vielleicht noch niemand, ausser Deyl, bei einer grösseren Anzahl von Fällen eingehend nach anatomischen Differenzen der beiden Hypophysishälften gesucht, und daher darf es uns nicht überraschen, dass die Literatur der Migräne in diesem Punkte sebr spärlich ist. Deyl ${ }^{32}$ ) gibt an, dass die Hypophysis manchmal lateralwärts verschoben oder dass sie derart asymmetrisch ist, dass die eine Hälfte derselben mehr entwickelt ist als die andere, und erblickt in diesem Umstande die Ursache für das einseitige Vorkommen der gewöhnlichen Migräne. Dass tatsächlich in dieser Gegend zahlreiche Anomalien vorkommen, ersehliesse ich auch aus der Sammelarbeit Sternbergs ${ }^{33}$ ), der behauptet, dass das Chiasma der Sehnerven nicht selten asymmetrisch liegt, so dass ihre intracraniellen Teile grosse Unterschiede in der Länge aufweisen. Auch die Zahl und die Lage der hypophysealen Arterien ist nach den Angaben Sternbergs keine einheitliche und sehr oft zweigt ausser den Ästchen, die von der Carotis innerhalb des Sinus cavernosus abgehen, noch ein Ästchen zur Hypophysis dort ab, wo die Carotis die Dura mater durchsetzt oder gleich dahinter.

Aber es ist schon im rorans wabrscheinlich, dass die anatomischen Differenzen, die die ophthalmoplegische Migräne bedingen, nicht stets von gleicher Art sein werden, wenn auch ihr Endeffekt, die Steigerung der normalen Hypophysisschwellung, stets der gleiche ist. Dies lässt

*) Nirgends findet sich in den betreffenden Krankengeschichten die Angabe, dass die Sehschärfe des ergriffenen Auges in einem Anfalle von ophthalmoplegischer Migräne abgenommen hätte. Auch in unserem Falle war der Visus sowohl im Anfalle als auch ausserhalb desselben stets gleich. In unserem Falle weist überdies der starke Astigmatismus auf eine angeborene Amblyopie hin. 
sich aus dem verschiedenartigen Beginn der ophthalmoplegischen Migräne erschliessen. Einmal beginnt dieselbe schon im zarten Alter, ja manchmal gleich nach der Geburt (Russel), das andere Mal erst im jugendlichen Alter; manchmal beginnt sie gleich als solche, manchmal geht jahrelang nur eine gewöhnliche (einseitige) Migräne ohne Iähmung voraus; manchmal entsteht die ophthalmoplegische Migräne nach einem Unfall (Karplus5)), ja sogar Tumoren der Hypophysis (Sternberg) oder Entzündungen in deren Umgebungen (Möbius ${ }^{1}$ )) können eine bestimmte Zeit hindurch eine symptomatische ophthalmoplegische Migräne hervorrufen. Möbius ${ }^{1}$ ) selbst gibt zu, dass auch im höheren Alter eine gewöhnliche idiopathische Migräne infolge verschiedener lokaler Veränderungen eine Oculomotoriuslähmung hervorrufen kann.

Da Sehstörugen bei der ophthalmoplegischen Migräne gewöhnlich fehlen, so scheint die Schwellung oder wenigstens ihr Maximum seitwärts von der Öffnung des Infundibulum, i. e. vom Chiasma zu liegen. Wir müssten demnach bei der ophthalmoplegischen Migräne eine noch grössere Dislokation oder Deformation der Hypophysis annehmen als Deyl bei der gewöhnlichen Migräne. Inwieweit diese Annahme mit der Wirklichkeit übereinstimmt, wird man erst dann nachzuweisen haben, wenn wiederum ein Fall von ophthalmoplegischer Migräne zur Sektion gelangen wird.

Es ist aber auch möglich, dass die Hypophysis sich an ihrem Platze befindet und dass der durch die Schwellung der asymmetrischen Hypophysis entstehende Druck nicht nach oben, auf das Chiasma oder den $N$. opticus, wirken kann, sondern nur nach der Seite in der Richtung auf den Sinus cavernosus. Bei verschiedenen klinischen Sektionen habe ich oft beobachtet, dass die Öffnung für das Infundibulum cerebri in der Dura mater oberbalb der Sella turcica nicht immer gleich weit ist. Manchmal ist sie kaum einige Millimeter weit und die Dura selbst endet mit einem unnachgiebigen Ring (manchmal wiederum ist dieser Ring schwächer und sein Lumen breiter als $1 \mathrm{~cm}$, so dass die Hypophysis zum grösseren Teil entblösst ist und fast umittelbar dem Chiasma und dem $\mathrm{N}$. opticus anliegt).

Welche von beiden Erklärungen die richtige ist, oder ob beide irgend einer dritten Erklärung weichen müssen, lässt sich ohne neue Sektionsbefunde der ophthalmoplegischen Migräne nicht konstatieren. Ich habe mir nur zum Zweck der Vollständigkeit anzudeuten erlaubt, worin etwa die Differenzen zwischen der ophthalmoplegischen und gewöhnlichen Migräne beruhen könnten.

Da nun bei der ophthalmoplegischen Migräne so häufig Symptome einer Venostase im Gebiet des Sinus cavernosus gefunden wurden, 
möchte ich es für wahrscheinlich halten, dass der Anfall von ophthalmoplegischer Migräne auf einer venostatischen Hyperämie beruht, wenn er auch ursprünglich mit einer aktiven Hyperämie der Hypophysis beginnt. Da die hypophysealen Venen in den Sinus cavernosus oder dessen nächste Verzweigungen münden, muss "die Hypophysis selbst unter der Venostase leiden, so dass wir hier analoge Verhältnisse vor uns bätten wie bei der Schwellung der Corpora cavernosa penis oder bei der Menstruation im retrovertierten Uterus. In einem Organ, das bei ungenügendem venösen Abfluss aktiv hyperämisch ist, muss eine um so grössere Schwellung auftreten und hierdurch liesse sich zugleich die Heftigkeit und die lange Dauer der Schmerzen bei den Anfällen der ophthalmoplegischen Migräne erklären. Unter diesen Umständen bleibt einerseits die Hypophysisschwellung an und für sich lange bestehen, andererseits kann die Ursache, ans welcher der Anfall mit der aktiven Hyperämie begann, nicht bald beseitigt werden, weil die Funktion der Drüse, die diese Ursache beseitigen soll, gestört, verlangsamt ist.

Mit Hilfe der Venostase können wir uns leicht vorstellen, inwiefern eine stationäre anatomische Disposition zur Entstehung der ophthalmoplegischen Migräne, d. i. eigentlich zur Steigerung der normalen Hypophysisschwellung, beitragen könnte Dass ein unter abnormen anatomischen Verhältnissen befindliches Organ schon bei der kleinen Schwellung sich selbst und eventuell auch seiner Umgebung den venösen Kreislauf verstopfen kann, lässt sich nicht bestreiten. 'Je mehr die lokale Disposition entwickelt wäre, eine desto k]einere Schwellung der Hypophysis würde zur Verringerung ihres venösen Abflusses resp. zur Entstehung eines Anfalls der ophthalmoplegischen Migräne genügen. Bei zufälliger Kombination der lokalen Disposition mit idiopathischer Migräne, bei welcher die Schwellung der Hypophysis vielleicht schon an und für sich eine grosse ist, müssten die Anfälle der ophthalmoplegischen Migräne besonders oft wiederkehren and stark hervortreten.

Da ich nun die ophthalmoplegische Migräne als eine lokale Erkrankung an der Basis cranii bezeichnet habe, könnte es vielleicht den Anschein erwecken, als ob ich mich hierdurch von der ursprünglichen Auffassung dieser Affektion als echte Migräne entfernt hätte. Die Mehrzahl der Autoren fasst die Migräne als eine cerebrale Erkrankung auf, die eventuell in einer allgemeinen Antointoxikation ihren Ursprung hat.

Die Erbringung des Beweises, dass auch die echte (gewöhnliche, idiopathische) Migräne in letzter Linie eigentlich auch nur eine basale und lokale Erkrankung ist, gehört zwar nicht direkt in den 
Bereich dieser Abhandlung; aber ich bin dennoch genötigt, mit einigen Worten meine Ansicht zu verteidigen, damit die echte hemikranische Natur der ophthalmoplegischen Migräne besser hervortrete.

Dass auch die gewöhnliche Migräne, resp. ihr wichtigstes, oft, auch einziges Symptom: der Kopfschmerz, einen basalen Ursprung haben könnte, lässt sich auch aus dem Umstand erschliessen, dass die Augenbindehant auf der Seite der Migräne oft hyperämisch ist, dass Tränenträufeln auf derselben Seite auftritt und die Schmerzen sich bei Bewegungen des Bulbus vergrössern (Möbius). Gewiss steigt bei Bewegungen der Augenmuskeln der Druck im Sinus cavernosus und infolge dessen nimmt wohl auch der Schmerz zu.

Auch die subjektive Angabe der Migränekranken, dass sie den Schmerz in der Tiefe der Augenhöhle (eventuell im Auge selbst) oder in der betreffenden Stirnhälfte, oft auch in der Schläfe derselben Seite empfinden, weist darauf hin, dass die Ursache der Schmerzen an der Gehirnbasis liegt.

Übrigens erklären auch jene Autoren, welche die Migräne als eine cerebrale Erkrankung auffassen, die Entstehung der Schmerzen extracerebral, indem diese Autoren zumeist an eine Reizung der meningealen Nerven denken (Gowers, Möbius, Edinger u. a.). Ja, bei der ophthalmoplegischen Migräne lokalisieren Charcot, Möbius, Karplus, Molou ${ }^{27}$ ) und Kollarits ${ }^{38}$ ) u. a. nicht allein die Ursache der Lähmung, sondern auch die Ursache der Schmerzen direkt auf die Basis cranii, in den Verlauf des N. oculomotorius, d. i. in die nächste Nachbarschaft des Sinus cavernosus.

Da sich an jenen Stellen, wo der Migräneschmerz vorwiegend gefühlt wird, der N. trigeminus verzweigt, stellte sich die Mehrzahl der Autoren bis jetzt den Migräneschmerz als eine Reizung des N. trigeminus vor. Ganz besonders denken an den N. trigeminus jene Autoren, die die Ursache der ophthalmoplegischen Migräne an dex Basis cranii suchen.

Dieser Erklärung der Entstehung des Migräneschmerzes im $\mathrm{N}$. trigeminus steht aber die klinische Erfahrung im Wege, dass sich die Trigeminusneuralgie von dem Migräneschmerz wesentlich unterscheidet. Unter anderem wird angeführt, dass der neuralgische Schmerz von den Kranken stets genan in den Verlauf des bertreffenden Nerven lokalisiert wird, während die Grenzen der Migräneschmerzen mehr diffus sind, wenn sich auch ihr Zentrum bestimmen lässt. Die Migräne ist stets mit Appetitlosigkeit, Magenbeschwerden oder gar mit Erbrechen verbunden. Die Neuralgie lässt diese Symptome vollkommen vermissen. Solange die Migräne nicht übermässig heftig ist, nimmt sie bei geistiger Arbeit an Intensität ab (namentlich wenn diese Arbeit nicht 
unangenehm, anstrengend ist); auch der Schlaf und das Essen kürzen die Migräne manchmal ab oder unterdrücken sie ganz und gar.

Auf Grund dieser qualitativen Symptome müssten wir eine Neuralgie auch dort diagnostizieren, wo dieselbe etwa durch eine periodisch exazerbierende Ursache hervorgerufen würde, so dass sie den Verlauf der Migräne imitieren würde.

Möbius und andere Autoren, welche den Migräneschmerz in einer Trigeminusreizung suchen, sprechen zwar von einer Reizung der meningealen Trigeminusfasern; damit ist aber der Unterschied zwischen der Neuralgie und der Migräne nicht erklärt, denn es ist nicht einzusehen, warum die Reizung der meningealen Fasern andere physiologische Eigenschaften haben sollte, als die Reizung der übrigen Fasern desselben Nerven. Dieser Widerspruch fällt Möbius selbst auf (l. c. Seite 104), aber er vermag ihn nicht befriedigend zu erklären. Zwar meint er, man könnte sich den Kopfschmerz und das Erbrechen bei der Migräne ebenso wie dieselben Symptome bei den groben meningealen Erkrankungen vorstellen, gibt aber zu, dass diese Analogie keine vollständige sei, und führt diesbezüglich selbst an, dass bei den groben Erkrankungen des Gehirns das Erbrechen gewöhnlich ohne vorangehendes Unbehagen eintrete; ausserdem erinnert er gleich Wernicke daran, dass das Erbrechen den Tumorschmerz keineswegs lindert. Dagegen bestehe bei der Migräne während der ganzen Dauer des Anfalls ein grösseres oder kleineres Unwohlsein seitens des Magens und Appetitlosigkeit; nach dem Erbrechen, ja manchmal nach blosser Ruhe lasse der Migräneschmerz gewöhnlich nach.

Übrigens ist es überhaupt fraglich, ob eine einfache lokale Hyperämie der Dura, wie sie Möbius bei der periodischen Oculomotoriuslähmung annimmt, einen so heftigen Schmerz und ein so hartnäckiges Erbrechen, wie sie bei der ophthalmoplegischen Migräne vorkommen, hervorzurufen imstande ist. Die klinische Erfahrung lehrt, dass Hirntumoren und intracerebrale Entzündungen viel eher Erbrechen hervorrufen als die rein meningealen Prozesse. Die meningealen, speziell die duralen Prozesse verursachen nur dann Erbrechen, wenn sie intensiver und ausgebreiteter sind, d. h. wenn auch schon das Gehirn selbst oder wenigstens die Sekretion der Cerebrospinalflüssigkeit leidet, resp. sich ändert.

Eine hochgradige Hyperämie der Gehirnhäute, i. e. eine wesentlichere Alteration der Cerebrospinalfüssigkeit, die notwendigerweise daraus resultieren müsste, ist auf Grund der Lumbalpunktion, welche de Lapersonne ${ }^{11}$ ) and Lafon mit Willemonte ${ }^{11}$ ) in typischen Fällen von ophthalmoplegischer Migräne ausgeführt haben, ebenfalls 
auszuschliessen. In beiden Fällen war die Cerebrospinalflüssigkeit makroskopisch und cytologisch unverändert.

Dass die Reizung der Dura allein zur Auslösung des Erbrechens nicht genügt, lässt sich auch aus dem Umstande erschliessen, dass eine Reizung der Rückenmarkshäute kein Erbrechen zur Folge hat, obwohl der Schmerz (z. B. bei Tumoren) ein sehr bedeutender zu sein pflegt.

Wenn wir in der Differentialdiagnose zwischen Trigeminusneuralgie und Migräne konsequent bleiben wollen, dann müssen wir die Erklärung des Migräneschmerzes durch eine Reizung der duralen oder peripheren Äste des Trigeminus vollkommen fallen lassen.

Da wir a priori schliessen können, dass der Sitz des Schmerzes bei der ophthalmoplegischen Migräne nicht weit vom Sitz der Lähmung entfernt sein dürfte, entsteht jetzt die Frage, ob sich in der Gegend des Sinus cavernosus noch ein anderer Nerv befindet, dessen Reizung den Anfall der ophthalmoplegischen Migräne und daher der Migräne überhaupt erklären könnte. Diese Frage muss bejaht werden, und zwar ist es noch der Sympathicus, der hier ein dichtes, unter dem Namen des Plexus caroticus, resp. cavernosus bekanntes Geflecht bildet. Diese beiden Geflechte hängen mit einander eng zusammen und deswegen nehmen wir von unserem Standpunkte aus auf beide in gleicher Weise Rücksicht.

Dass an dem Migräneschmerz auch sympathische Nerven beteiligt sein könnten, vermutete bereits du Bois-Raymond ${ }^{39}$ ) und beschuldigte diesbezüglich einen Spasmus der glatten Muskulatur der Gehirngefässe nach Analogie jener kolikartigen Schmerzen, die bei einem ähnlichen Spasmus der glatten Muskulatur des Uterus oder Darms entstehen. Diese Erklärung passte aber nur für die sog. vasokonstriktorische Form der Migräne und wurde gegenstandslos, als es sich zeigte, dass man die Migräne nicht als eine Anämie des Zentralnervensystems auffassen könne.

Auch Edinger ${ }^{25}$ ) führt in seiner Erklärung der Migräne eine Reizung der meningealen Vago-Sympathicusfasern an und will auf diese Weise das Erbrechen und das Übelbefinden erklären, während er die Schmerzen auf eine Reizung der Trigeminusfasern zurückführt. Diese Vermutung entfällt aber aus denselben Gründen, nach denen eine jede meningeale Reizung bei der Migräne unwahrscheinlich ist.

Dass tatsächlich im sympathischen Nervensystem Schmerzen entstehen und Neuralgien vorkommen können, ist allgemein bekannt, so dass ich nicht näher darauf eingehen muss; es bandelt sich nur darum, ob der sympathische Schmerz im Plexus caroticus durch seine 
Qualität den Schmerz bei der Migräne überhaupt und bei der ophthalmoplegischen Migräne insbesondere zu erklären vermag.

Das Zentrum und die Ausstrahlungsrichtung des Migräneschmerzes stimmen mit der Lokalisation und der Verästelung des sympathischen Basalgeflechts gut überein. Es ist bekannt, dass aus dem basalen Sympathicusgeflecht ein grosser Teil der Fasern in das Auge derselben Seite geht und dass dasselbe Geflecht Ästchen oder kleinere Geflechte längs aller cerebraler und meningealer Gefässe (Arterien, Venen und Sinus) entsendet, so dass es begreiflich ist, dass ein Schmerz, dessen Zentrum in der Tiefe der Orbita, i. e. im Plexus cavernosus oder caroticus liegt, im Auge, in der Stirn und, wenn er gar zu stark wird, eventuell auch im Hinterhaupt empfunden wird. In der Schläfengegend wird der Schmerz vielleicht aus dem Grunde so häufig angegeben, weil das basale Sympathicusgeflecht hier der Schädeloberfläche relativ am nächsten liegt. In jenen Fällen, wo der Schmerz ausschliesslich nur im Auge empfunden wird, wird vielleicht der Augenteil des basalen Sympathicusgeflechtes am meisten beteiligt sein.

In derselben Weise wie der Migräneschmerz sind alle Sympathicusschmerzen charakterisiert, mögen sie in welchem Gebiet immer entstehen. Bei Gallen- und Nierensteinkoliken, bei den verschiedenen Koliken und Neuralgien des Bauch- oder Brustsympathicus gibt der Kranke nur die Stelle, wo das Maximum des Schmerzes sitzt oder wohin der Schmerz vorwiegend ausstrahlt, gut an, vermag aber nicht den Schmerz so, wie bei den Neuralgien der Cerebrospinalnerven, genauer zu lokalisieren oder begrenzen. Interessant ist ferner, dass bei allen Sympathicusschmerzen, wenn dieselben einen gewissen Grad erreichen, Erbrechen auftritt und zwar ohne Rücksicht darauf, in welcher" Partie des ganzen Sympathicus der Schmerz sitzt. So z. B. tritt Erbrechen ein: bei Anfällen von. Chole- und Nephrolithiasis, und zwar bevor noch das Bauchfell durch sekundäre Veränderungen gereizt ist; übrigens erfolgt das Erbrechen im Stadium der grössten Schmerzen auch nach Traumen der Hoden und beim Glaukom*), was sich nur durch Reizung der in die Hoden und ins Auge in reichlicher Menge eintretenden sympathischen Fasern erklären lässt; ja, auch der Schmerz bei Angina pectoris strablt oft in den Bauch aus und die Kranken geben in solchen Fällen manchmal an, dass ihnen übel vom Magen sei (Maixner $\left.{ }^{40}\right)$ ), obzwar der anginöse Anfall nur kurze Zeit dauert.

Durch die Lokalisation der Migräneschmerzen in den Plexus caroticus, eventuell cavernosus kann man auch die Steigerung der

*) Möbius und Schmidt-Rimpler bemerken, dass der Glaukomanfall durch seine ganze Qualität der Migräne sehr ähnlich sei. 
Schmerzen nach dem Neigen des Kopfes und andererseits ein Nachlassen derselben bei Kompression der Arteria carotis derselben Seite erklären. Die Spannung, welche die Ursache dieser Schmerzen ist, nimmt im ersten Fall zu und im anderen Fall ab.

Wilbrand und Saenger ${ }^{41}$ ) bestätigen die Erfahrungen Oppenhejms, dass Leute, die dauernd an einseitiger Migräne leiden, auf derselben Seite eine verengte Augenspalte und Pupille haben, was für eine Sympathicuslähmung spreche. Dass bei einem gewöhnlichen Migräneanfall die gleichseitige Pupille verengt zu sein pflegt, gibt auch Möbius ${ }^{34}$ ) mit Rücksicht auf die Angaben anderer Autoren zu. Er hat die Verengerung der Augenspalte auf der Seite der Schmerzen im Migräneanfall öfters beobachtet, erklärt dieselbe aber als einen unwillkürlichen Lidschluss zum Lichtschutz.

Oft wurden auch im Anfall einer gewöbnlichen sowie auch ophthalmoplegischen Migräne vasomotorische oder sekretorische Störungen beobachtet. Die vasomotorischen Veränderungen (im Gesicht, im Auge usw.) haben, wie neuere Erfahrungen lehren, nichts Konstantes an sich; ihre Form und Ausbreitung richten sich nach der Individualität und ändern sich manchmal sogar während des Anfalls. Von den sekretorischen Veränderungen ist als sehr häufige Erscheinung ein höherer Säuregehalt des Magensaftes und Tränenträufeln auf jener Seite, wo der Schmerz ist, hervorzuheben; seltener sind Speichelfluss, Schweisse, Polyurie u. dgl. Alle diese Veränderungen lassen sich entweder als direkte Wirkung einer lokalen Reizung des basalen Sympathicusgeflechtes oder als Fernwirkung, eventuell als Reflexwirkung erklären. Diese Erklärung ist durchaus nicht unnatürlich, weil die vasomotorischen und sekretorischen Funktionen vorwiegend in den sympathischen Geflechten und Fasern ihren Sitz haben.

Durch Verlegung des Migräneschmerzes aus dem N. trigeminus in den Sympathicus können wir uns also den ganzen Migräneanfall in allen seinen Erscheinungen leicht erklären. Dureh denselben Vorgang wird zugleich auch die Hypothese wahrscheinlicher, dass der Migräneanfall mit einer periodischen Schwellung der Hypophysis eng zusammenhängt. Das sympathische Carotisgeflecht liegt in unmittelbarer Nachbarschaft der Hypophysis und muss daher bei einer Schwellung dieser Drüse von allen basalen Nerven in erster Reihe in Mitleidenschaft gezogen werden, und daher beginnt auch ein jeder Anfall von ophthalmoplegischer Migräne mit Schmerzen, während sich die Lähmung erst später hinzugesellt, wenn vielleicht infolge Venostase die Schwellung der Hypophysis, resp. der subdurale Druck noch mehr zugenommen hat.

Wenn die Autoren die echte (idiopathische) Migräne für eine 
cerebrale Erkrankung erklären, so lassen sie sich hierbei hauptsächlich durch die Erklärung der Aura leiten, wobei stets in erster Linie an eine visuelle Aura gedacht wird. Die Schwellung der Hypophysis schliesst, wie ich bereits früher erwähnt habe, eine Gesichtsaura keineswegs aus, sondern vermag dieselbe in gegebenen Fällen peripherisch zu erklären.

Bekanntlich kann die Aura, mit der ein Migräneanfall beginnt, von verschiedener Art sein, beginnend von einem einfachen diffusen szintillierenden Skotom bis zu sehr komplizierten Formen: Teichopsie, Parästhesie der Extremitäten, totalem Verlust der Sprache oder verschiedenen Störungen derselben (Verlust der Kenntnis fremder Sprachen, oder einer gewissen Anzahl von Ausdrücken, reine Agraphie u. dgl.), ja sogar bis zu den mannigfachsten psychischen Störungen. Diese Erscheinungen kann eine einfache Hypophysisschwellung allerdings nicht erklären.

Andererseits ist es aber überhaupt fraglich, ob solche komplizierte Aurae noch zur echten Migräne gehören, und ob es sich hier nicht um eine andere Krankheit, um Epilepsie, handelt. Jedenfalls ist es auffallend, dass bei einer stark entwickelten Aura der Schmerzanfall ein schwacher und kurzdauernder zu sein pflegt oder ganz und gar fehlen kann (Möbiu s $\left.{ }^{42}\right)$ ). Solche Anfälle mit stark entwickelter Aura können wir leicht als epileptische auffassen, denen ein stärkerer oder leichterer Anfall von echter Migräne nachfolgt.

Der Migräneanfall stellt sich gern infolge irgend einer zufälligen Ursache ein, wobei psychische oder nervöse Exzesse, zu denen wir gewiss auch den epileptischen Anfall zählen können, eine nicht unbedeutende Rolle spielen. Auch ist es bekannt, dass der epileptische Anfall ohne Rücksicht auf seine Form von Kopfschmerzen gefolgt zu sein pflegt.

Zwischen Aura und Kopfschmerz besteht kein bestimmter konstanter Zusammenhang. Die Aura begleitet nicht einen jeden Migräneanfall und tritt gewöhnlich nur während eines bestimmten Lebensalters auf. Mancher Patient leidet an Anfällen von Aura und von eigentlicher Migräne (Kopfschmerzen), die zeitlich vollkommen getrennt sind (migraine dissociée der französischen Autoren). Die Aura und der Kopfschmerz bei Migräne sind zwar einseitig, aber die Seite des einen und des anderen Symptoms schwankt; bald ist der Schmerz auf derselben Seite wie die Aura, bald aber auf der anderen. Gowers und Möbius gestehen ein, dass dieses Missverhältnis auffallend sei, und vermögen es nicht zu erklären. Suchmidt-Rimpler erklärt direkt, er könne das szintillierende Skotom nicht so ohne weiteres als einen Bestandteil der Migräne auffassen. 
Féré ${ }^{43}$ ) trennt die ophthalmische Migräne vollständig von der gewöhnlichen Migräne und hält sie für ein Äquivalent des Petit mal, für eine partielle sensorielle Epilepsie. Möbius opponiert zwar dieser Ansicht, gibt aber selbst zu, dass die Epilepsie auch unter dem Bilde der "ophthalmischen Migräne" verlaufen könne, besonders wenn diese auch von Parästhesien und Aphasie begleitet ist, so dass die Differentialdiagnose unmöglich wird. Übrigens wurde von verschiedenen Autoren eine ganze Reihe von Fällen beschrieben, in denen sich aus der "ophthalmischen Migräne" später eine sichere Epilepsie entwickelte (Gowers, Mingazzini, Pelnàr 44), Repka45) u. a. m.), indem z. B. dieselbe visuelle Aura, die den Migräneanfällen voranging, auch vor den epileptischen Anfällen auftrat. Auffallend ist es auch, dass das Bromkali, das wirksamste Medikament bei der Epilepsie, sich nur bei der ophthalmischen und nicht bei der gewöhnlichen Migräne bewährt, was nicht bloss Charcot und Féré konstatierten, sondern auch Möbius.

Ich will aber nicht sagen, dass eine jede visuelle Aura ausnahmslos auf Epilepsie hinweist. Berechtigt sind wir zu einer solchen Vermutung nur bei jenen Fällen, die eine komplizierte, mit Bestimmtheit auf einen cortikalen Ursprung hindeutende Aura haben. Dagegen können wir eine einfache visuelle Aura auch peripher erklären. Die Grenze zwischen einer epileptischen und einer echten hemikranischen Aura lässt sich bis jetzt noch nicht mit Bestimmtheit ziehen, aber vielleicht werden unsere klinischen Kriterien mit der Zeit vollkommener werden. Monoculäre Skotome oder Skotome, die beide Gesichtsfeldhälften einnehmen oder deren Seite mit der Seite der Migräne in einem konstanten Verhältnis steht, könnten mit einer Schwellung der Hypophysis zusammenhängen.

Es ist bekannt, dass die echte Migräne, auch wenn sie sich das ganze Leben hindurch in häufigen and schweren Anfällen wiederholt, unschädlich ist. Diese Benignität werden wir um so eher begreifen, wenn wir die Migräne als eine basale und nicht als eine cerebrale (cortikale), nach Art der Epilepsie sich abspielende Erkrankung auffassen (Liveing, Gowers, Möbius). Die Epilepsie schädigt oft die Intelligenz, während die Migräne sie intakt lässt. Aus diesem Grunde ist Spitzers cerebrale Erklärung der Migräne um so unwahrscheinlicher, als hier ziemlich grobe Prozesse in der einen oder anderen Hemisphäre angenommen werden, die nicht so ohne weiteres während des ganzen Lebens ohne Folgen bleiben könnten.

Übrigens ist, wenn wir die Migräne als eine periodisch wiederkehrende Hyperämie und Schwellung der Hypophysis auffassen, die Migräne hiermit nicht als eine rein periphere Erkrankung hingestellt. 
Diese Schwellung ist wohl die Reaktion auf eine mehr oder weniger allgemeine Autointoxikation, bei der eine vasomotorische und sekretorische Reizung höchstwahrscheinlich eine wichtige Rolle spielt. Aus diesen Gründen bleibt auch die neuropathische Bedeutung der Migräne unberührt, weil die Antointoxikation sowie auch die Änderung der vasomotorischen Reaktion in der Neuropathologie oft eine Rolle spielen.

Die Sehwellung der Hypophysis ist das letzte Glied in der Kette der die Migräne bedingenden vitalen Erscheinungen, es ist aber das charakteristischste, weil durch dieselbe der Migräneanfall eigentlich hervorgerufen wird. $O b$ nun diese Schwellung bei der einfachen Migräne ein rein altiver Prozess ist, oder ob sie bei der ophthalmoplegischen Migräne mehr passiv, durch eine lokale anatomische, sei es nun eine angeborene oder erworbene Disposition verursacht wird, ist nicht entscheidend. In beiden Fällen handelt es sich im Grunde um denselben Prozess, i. e. um eine Schwellung der Hypophysis, die nach bestimmten autoregulativen Grundsätzen erfolgt, unter deren Einfluss die Migräne einen bestimmten klinischen Verlauf nimmt, und dieses ist beiden Formen der Migräne - der einfachen und der ophthalmoplegischen - gemeinschaftlich.

Wenn ich meine Ansicht über die ophthalmoplegische Migräne kurz zusammenfassen soll, so kann dies folgendermassen geschehen:

Ich glaube, dass die Grundlage der ophthalmoplegischen Migräne die echte Migräne ist, und dass diese Migräne die Ursache der Oculomotoriuslähmung ist, oder anders gesagt, der Begriff der ophthalmoplegischen Migräne reicht so weit, als die Ursache der Oculomotoriuslähmung eine echte, sei es nun idiopathische oder symptomatische, Migräne ist.

Sowohl die gewöhnliche als auch die ophthalmoplegische Migräne sind in ihrem Endeffekt eine basale, lokale Erkrankung, und zwar handelt es sich wahrscheinlich um eine periodische Schwellung der Hypophysis, die von besonderen autoregulativen vasomotorischen Einflïssen beherrscht wird. Bei der einfachen Migräne ist diese Schwellung eine allgemeine oder einseitige, und kann in letzterem Falle die Seite wechseln; bei der ophthalmoplegischen Migräne ist sie stets eine einseitige, was vielleicht durch eine laterale Dislokation oder Deformation der Hypophysis bedingt ist.

Während bei der gewöhnlichen Migräne die annormale Schwellung der Hypophysis, nach meiner Anschaung, einen rein aktiven Prozess (Hyperämie) darstellt, welcher infolge einer neuropathischen Grundlage zum Ausdruck kommt, beruht die Schwellung bei der ophthalmoplegischen Migräne hauptsächlich auf einer Venostase der Hypophysis, 
wenn anch der Anfall mit einer kleinen aktiven (von Zeit zu Zeit auch beim Gesunden wiederkehrenden) Schwellung der Hypophysis beginnt. Diese Venostase, obzwar ihre unmittelbare Ursache in einer spontanen Sehwellung der Hypophysis liegt, ist zugleich als Folge der erwähnten Dislokation oder Deformation der Hypophysis, resp. einer anderen lokalen Disposition zu betrachten.

Da die ophthalmoplegische Migräne auf einer besonderen lokalen anatomischen, angeborenen oder erworbenen Disposition beruht, muss sie nicht erblich sein wie die gewöhnliche Migräne, kann aber schon bald nach der Geburt beginnen oder aber auch erst später erworben werden. Ohne die anatomische Disposition kann die gewöhnliche Migräne in die ophthalmoplegische nicht übergehen.

Durch die Hypophysisschwellung entsteht ein Druck auf die Umgebung und dadurch leidet in erster Reihe der Sympathicus, was sich durch Schmerz und Erbrechen äussert, und in zweiter Reihe wenn die lokale Disposition besteht - auch der N. oculomotorius, was als periodische Lähmung in die Ercheinung tritt. Wie die Beschädigung des Oculomotorius zustande kommt, lässt sich nicht sicher entscheiden, es ist aber wahrscheinlich, dass die Drnckwirkung der geschwollenen Hypophysis, infolge der lokalen Disposition, mehr seitlich verschoben ist, wodurch die Dura mater, welche hier über die Gegend des Sinus cavernosus eine Art von Gewölbe macht, gespannt und dadurch wieder der Oculomotorius in seinem Durchtritt eingeklemmt wird. Weil die Druckwirkung der geschwollenen Hypophysis seitlich verschoben ist, bleibt das Chiasma vom Drucke unversehrt, und deshalb ist die visuelle Aura bei der ophthalmoplegischen Migräne so selten.

Dem hochgeehrten Herrn Prof. Dr. E. Maixner, der meine Aufmerksamkeit auf diese interessante Krankbeit gelenkt und mir bei- der Untersuchung unseres Falles wertvolle Winke gegeben hat, statte ich meinen höflichsten Dank ab.

\section{Literatur.}

1) Möbiu s, Deutsche Zeitschrift f. Nervenheilkde. 1900. Bd. 17. \$. 294.

2) Charcot (resp. Guinon), Clinique d. mal. du système nerveux. Paris 1892. T. I. p. 70.

3) Vissering, Münch. med. Wochenschr. 1889. Nr. 41.

4) Oppenheim, Lehrbuch der Nervenkrankh. 1902. S. 423.

5) Karplus, Jahrb. f. Psych. u. Neurol. 1902. Bd. 22. S. 158.

6) Seiffer, Berliner klin. Wochenschr. 1900. S. 657. 
7) Schmidt-Rimpler, Nothnagels spez. Path. u. Ther. 1898. Bd. 21. S. 167.

8) Bernheimer, Gräfe-Sämischs Handbuch der ges. Augenheilk. 2.', Aufl. 39. Lief. 1902 . S. 87.

9) Wilbrand u. Saenger, Neurologie d. Auges. Wiesbaden 1900. 1. Bd. S. 483.

10) Ryba, Sborník klinický. Bd. 4. S. 391. (2. Fall.)

11) Leclézio, La migraine ophthalmoplégique. Thèse. Bordeaux 1905.

12) 1. c. 1. Fall.

13) Möbius, Migräne. Nothnagels spez. Pathol. u. Therapie. 1894. Bd. 12, Heft 2. S. 82.

14) Bernhardt, Berl. klin. Wochenschr. 1889. S. 1010.

15) Joachim, Archiv f. klin. Medizin. 1889. Bd. 44. S. 185.

16) Gowers, Handbuch der Nervenkrankheiten. 1892. 3. Bd. S. 234.

17) Haltau, Jahrb. f. Psych. u. Neurol. 1901. Nr. 20.

18) Senator, Zeitschr. f. klin. Medizin. 1888. Bd. 13. S. 252.

19) Paderstein, Schmidts Jahrbücher. Bd. 265. S. 31.

20) Manz, Berliner klin. Wochenschr. 1889. S. 749.

21) Derselbe, Ebenda. 1885. S. 637.

22) Ballet, Schmidts Jahrbücher. Bd. 252. S. 23. (1896.)

23) Seeligmüller, Eulenburgs Enzyklop. 1897. Bd. 15. S. 282.

24) Charcot, Lecons du mardi. 1892. T. 1. p. 22.

25) Edinger, Deutsche Klinik. 1906. Bd. 6. 1. Abt. S. 83.

26) Thomayer, Ophthalmische Migräne. Ćas. lék. cesk. 1894 (böhm.).

27) Molon, Revue neurolog. 1904. S. 243.

28) Richter, Archiv f. Psych. u. Nervenkrankh. 1887. Bd. 18. S. 259.

29) Karplus, Wiener klin. Wochenschr. 1895. Nr. 50. 2. Fall.

30) Weiss, Wiener med. Wochenschr. 1885. S. 522.

31) Luzenberger, Neurolog. Zentralbl, 1893. Bd. 17. S. 73.

32) Deyl, Explication anatomique de la migraine. Communic. provis. au Congrès internat. à Paris. 1900.

33) Sternberg, Akromegalie. Nothnagels Path. u. Ther. 1903. Bd. 7, H. 2.

34) Möbius, Migräne l. c. S. 26.

35) Schmidt-Rimpler, l, c. S. 107 u. 283.

36) Jolly, Berl. klin. Wochensehr. 1902. Nr. 42-43.

37) Schilling, Münch. med. Wochenschr. 1902. Nr. 18.

38) Kollarits, Deut. Zeitschrift f. Nervenheilk. 1904. Bd. 26. S. 128.

39) Du Bois-Raymond, Arch. f. Anat. u. Phys. 1860. S. 467.

40) Maixner, Časopis lékaři českých. 1905. S. 25.

41) Wilbrand u. Saenger, 1. c. S. 557.

42) Möbius, Migraine l. c. S. 48 u. 50.

43) Féré, Epilepsies et épileptiques. Paris 1890. S. 50.

44) Pelnàr̆, Thomayers Jubilejní sborník. 1901. S. 67.

45) Řepka, ebenda S. 85. 